\title{
Theory and Experiment of Ultrahigh-Gain Gyrotron Traveling Wave Amplifier
}

\author{
Kwo Ray Chu, Fellow, IEEE, Han-Ying Chen, Chien-Lung Hung, Tsun-Hsu Chang, \\ Larry R. Barnett, Member, IEEE, Shih-Hung Chen, Tz-Te Yang, and Demosthenes J. Dialetis
}

(Invited Paper)

\begin{abstract}
Physics and technology issues of importance to the high-gain gyrotron traveling wave amplifier (gyro-TWT) are investigated in theory and experiment. The gyro-TWT is known to be highly susceptible to spurious oscillations, especially in high gain operations. In the current study, oscillations of various origins are classified and characterized with detailed theoretical modeling. They are shown to be intricately connected to the interplay between the absolute/convective instabilities, circuit losses, and reflective feedback. Knowledge of these processes leads to the concept of an ultra high gain scheme which employs distributed wall losses for the suppression of spurious oscillations. A proof-of-principle Ka-band gyro-TWT experiment stable at zero drive has produced $93 \mathrm{~kW}$ saturated peak power at $26.5 \%$ efficiency and $70 \mathrm{~dB}$ gain, with a $3 \mathrm{~dB}$ saturated output power bandwidth of $3 \mathrm{GHz}$. The saturated gain is more than $30 \mathrm{~dB}$ beyond that previously achieved.
\end{abstract}

Index Terms - High gain, millimeter wave amplifier, spurious oscillation.

\section{INTRODUCTION}

$\mathbf{H}$ IGH power and broad bandwidth capability of the gyrotron traveling wave amplifier (gyro-TWT) makes it an attractive source of coherent radiation in the millimeter wavelength range. Steady progress in theory and experiment has been made over an extended period of time. Achieved performances [1]-[17] of the gyro-TWT based on a weakly relativistic electron beam are summarized in Table I.

In contrast to conventional linear beam devices, the electron beam employed in the gyrotron possesses a transverse motion at the electron cyclotron frequency. It is this property that allows the beam to selectively interact with a high-order waveguide mode at a high cyclotron harmonic by properly matching the resonance conditions. However, the additional degree of freedom provided by the multitude of cyclotron harmonics can also generate numerous spurious oscillations. Fig. 1 plots the $\omega-k_{z}$ diagram of the transverse electric $\mathrm{TE}_{11}$ and $\mathrm{TE}_{21}$ waveguide modes (for a waveguide radius

Manuscript received October 9, 1998; revised December 16, 1998. This work was supported by the National Science Council, R.O.C.

K. R. Chu, H.-Y. Chen, C.-L. Hung, T.-H. Chang, and L. R. Barnett are with the Department of Physics, National Tsing Hua University, Hsinchu, Taiwan, R.O.C.

S.-H. Chen is with the National Center for High-Performance Computing, Hsinchu, Taiwan, R.O.C.

T.-T. Yang is with the Synchrotron Radiation Research Center, Hsinchu, Taiwan, R.O.C.

D. J. Dialetis is with the Science Applications International Corporation, McLean, VA 22102 USA.

Publisher Item Identifier S 0093-3813(99)03365-2. of $0.27 \mathrm{~cm})$ and the fundamental $(s=1)$ and second $(s=2)$ cyclotron harmonic beam-wave resonance lines. As is well understood, interactions in the backward wave region (points 1 and 2) are sources of absolute instabilities [18]-[24] (oscillations due to an internal feedback), whereas those in the forward wave region (points 3, 4, and 5) are normally, but not always, convective instabilities. The gyroTWT is a complicated case because it exploits a convective instability near the cutoff frequency (e.g., point 3) which turns into an absolute instability at sufficiently high beam current when the unstable spectrum extends into the backward wave region. These various absolute instabilities can easily be the dominant sources of oscillations in an unsevered interaction structure [Fig. 2(a)]. For example, in a fundamental harmonic gyro-TWT operating at the lowest order waveguide mode (point 3 in Fig. 1), a second harmonic absolute instability (point 2 in Fig. 1) has been observed [6] at beam current as low as $0.1 \mathrm{~A}$. The instability was shown to compete with and eventually be suppressed by the amplified wave, but linearity was affected at low drive powers. Interestingly, a harmonic gyro-TWT can be more stable against the absolute instability, as shown by theory [25] and demonstrated in experiment [14].

Feedback due to reflections at the input-output couplers and structural nonunifomities presents a different source of oscillations (referred to as the reflective oscillation) in the high-gain regime. Even when the gain is kept below the oscillation threshold, reflective feedback can still cause ripples in the gain and output power spectra.

Reflective oscillations are common in traveling wave structures. The feedback loop can be effectively eliminated by a sever as has been a standard practice in conventional TWT's. The absolute instability, a much more serious problem to the gyro-TWT than to the conventional TWT, is basically different from the reflective oscillation in that the backward wave associated with the absolute instability is internally generated by the ac electron beam current. In the experiment of [8], a sever was used as a remedy for the absolute instability [Fig. 2(b)]. But the two stages, though separated by the sever, are coupled by the beam. With the beam providing an internal path, the sever does not really quite separate the interaction structure into two isolated sections to produce a substantial stabilizing effect. With the sever, the start-oscillation current can be increased from 0.1 to $0.9 \mathrm{~A}$, but still well below what is required for high-power generation. 
TABLE I

Achieved Performance of Gyro-TWT's Based on a Weakly Relativistic Electron Beam

\begin{tabular}{lllccccccc}
\hline year & \multicolumn{1}{c}{$\begin{array}{c}\text { institution } \\
\text { (references) }\end{array}$} & $\begin{array}{c}\text { cyclotron } \\
\text { harmonic }\end{array}$ & $\begin{array}{c}\text { V } \\
(\mathrm{kV})\end{array}$ & $\begin{array}{c}\text { freq. } \\
(\mathrm{GHz})\end{array}$ & $\begin{array}{c}\text { 3dB } \\
\text { bandwidth } \\
(\%)\end{array}$ & $\begin{array}{c}\text { peak } \\
(\mathrm{kW})\end{array}$ & $\begin{array}{c}\text { saturated } \\
\text { gain } \\
(\mathrm{dB})\end{array}$ & $\begin{array}{c}\eta \\
(\%)\end{array}$ \\
\hline 1979 & NRL (Refs. 1 and 2) & 1 & 70 & 35 & 1.5 & 16.6 & 20 & 7.8 \\
1981 & Varian (Refs. 4 and 5) & 1 & 60 & 5 & 6 & 120 & 18 & 26 \\
1982 & Varian (Ref. 16) & 1 & 50 & 94 & 2 & 20 & 30 & 8 \\
1993 & NRL (Refs. 10 and 15) & 1 & 33 & 35 & 20 & 8 & 25 & 16 \\
1994 & UCLA and UC Davis & 2 & 80 & 16 & 2.1 & 207 & 16 & 13 \\
& (Refs. 11 and 14) & & & & & & & \\
1995 & NTHU (Refs. 6 and 13) & 1 & 100 & 35 & 12 & 62 & 33 & 21 \\
1998 & NTHU (Ref. 17 and this paper) & 1 & 100 & 35 & 8.6 & 93 & 70 & 26.5 \\
\hline
\end{tabular}

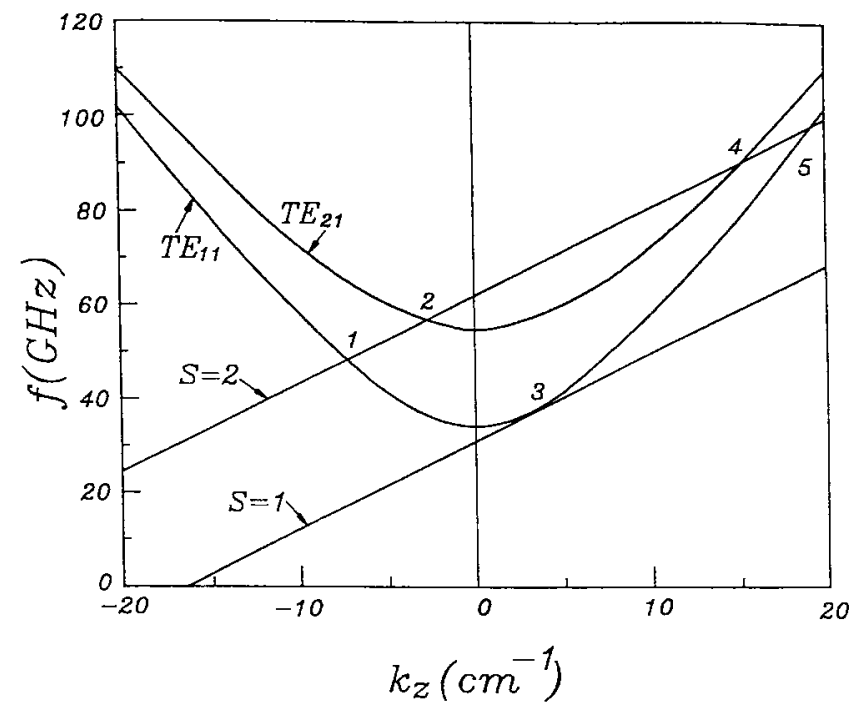

Fig. 1. $\omega-k_{z}$ diagram of a fundamental harmonic gyro-TWT operating in the $\mathrm{TE}_{11}$ mode (point 3). Other possible convective instabilities (points 4 and 5) and absolute instabilities (points 1 and 2) are also indicated.

Recently, an interaction structure with distributed wall losses was shown to be effective in suppressing both types of oscillations [13]. The losses are distributed over much of the linear interaction region [Fig. 2(c)]. Like the sever, the lossy section cuts off the path of the reflective feedback loop. In contrast to the sever, however, it is an integral part of the linear amplification stage. To the predominantly backward power flow of the absolute instability, the lossy section also functions as an effective energy sink. The absolute instability threshold can be raised to $26 \mathrm{~A}$ as a result [13].

The current paper presents theoretical and experimental studies of a high-gain gyro-TWT based on the distributedloss scheme. High gain operation enhances the possibility of spurious oscillations caused by various internal and external feedback mechanisms. Thus, the physics issues involved are the identification, characterization, and suppression of possible sources of oscillations.

Three types of oscillations have been analyzed theoretically. Each type of oscillation exhibits different characteristics. Experiments have been conducted to investigate these oscillations unsevered structure

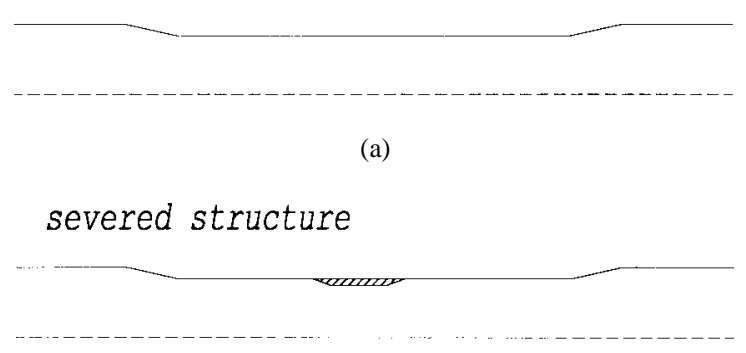

(b)

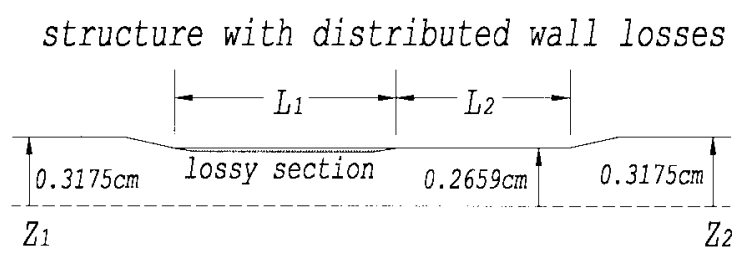

(c)

Fig. 2. Schematic drawings of the unsevered, severed, and distributed-loss gyro-TWT. Both ends of the uniform cross-section interaction structure are tapered for broad-band, side-wall input-output coupling at $z_{1}$ and $z_{2}$. Configuration (c) is studied in this paper.

and their stabilization by proper distribution of wall losses. Based on these results, a Ka-band gyro-TWT with $93 \mathrm{~kW}$ saturated peak output power at $70 \mathrm{~dB}$ stable gain, and $26.5 \%$ efficiency has been demonstrated in stable operation.

In Section II, equations for nonlinear computations are formulated with realistic modeling of wall losses and structural nonuniformities. In Section III, the basic concept of the high gain scheme is discussed and likely sources of oscillations are analyzed. In Section IV, we report results of the high-gain gyro-TWT experiment.

\section{A Formalism For Nonlinear CAlculations}

\section{A. Field Equations}

The wave equation for the radio frequency (RF) magnetic field $\vec{B}$ driven by of a current source can be written

$$
\nabla^{2} \vec{B}-\frac{1}{c^{2}} \frac{\partial^{2}}{\partial t^{2}} \vec{B}=-\frac{4 \pi}{c} \nabla \times \vec{J}
$$

where $\vec{J}$ is the beam current density. 
We simplify the problem by neglecting the beam effects on the transverse field profiles. The formal solution for a nonuniform waveguiding structure is normally in the form of eigen-mode expansion. If conditions of operation favor the generation of a particular mode and the structural nonuniformity is sufficiently weak such that mode conversion is of negligible importance to the problem under consideration, we may construct a single-mode solution by requiring that it be reducible to the known mode of a uniform waveguide and that it conserve the energy. Fields of the circularly polarized $\mathrm{TE}_{m n}$ mode can then be approximated by the real parts of

$$
\begin{aligned}
& B_{z}=k_{m n}^{2} f(z) J_{m}\left(k_{m n} r\right) e^{-i(\omega t-m \theta)} \\
& B_{r}=k_{m n} f^{\prime}(z) J_{m}^{\prime}\left(k_{m n} r\right) e^{-i(\omega t-m \theta)} \\
& B_{\theta}=\frac{i m}{r} f^{\prime}(z) J_{m}\left(k_{m n} r\right) e^{-i(\omega t-m \theta)} \\
& E_{r}=\frac{-\omega m}{c r} f(z) J_{m}\left(k_{m n} r\right) e^{-i(\omega t-m \theta)} \\
& E_{\theta}=\frac{-i \omega k_{m n}}{c} f(z) J_{m}^{\prime}\left(k_{m n} r\right) e^{-i(\omega t-m \theta)}
\end{aligned}
$$

where $k_{m n}=x_{m n} / r_{w}(z), r_{w}(z)$ is the wall radius as a function of the axial position $z, x_{m n}$ is the $n$th root of the derivative of Bessel function $J_{m}^{\prime}(x)$, and $f(z)$ is the field profile function along the axis. In (2), the transverse field profile is approximated by that of a uniform waveguide, while the axial field profile $f(z)$ is to be evaluated self-consistently from the wave equation (1) and equations of motion of the electrons. We shall restrict our consideration to the quasi steady-state by assuming that the wave frequency $\omega$ is real.

Substituting (2a) into the $z$-component of (1) and neglecting terms involving derivatives of $r_{w}(z)$, we obtain

$$
\begin{aligned}
\operatorname{Re}\left[k_{m n}^{2}\left(\frac{\partial^{2}}{\partial z^{2}}+k_{z}^{2}\right)\right. & \left.f(z) J_{m}\left(k_{m n} r\right) e^{-i(\omega t-m \theta)}\right] \\
& =-\frac{4 \pi}{c} \frac{1}{r}\left[\frac{\partial}{\partial r}\left(r J_{\theta}\right)-\frac{\partial}{\partial \theta} J_{r}\right]
\end{aligned}
$$

where $k_{z}^{2}$ is defined as

$$
k_{z}^{2}=\left(\omega^{2}-\omega_{c m n}^{2}\right) / c^{2}
$$

and $\omega_{c m n}=k_{m n} c$ is the local cutoff frequency. If wall losses are included, (3) remains unchanged with $k_{z}^{2}$ modified by a loss factor (in the brackets of the following equation):

$$
\begin{aligned}
k_{z}^{2}= & \frac{1}{c^{2}}\left\{\omega^{2}-\omega_{c m n}^{2}\left[1-(1+i) \frac{\delta}{r_{w}}\right.\right. \\
& \left.\left.\cdot\left(1+\frac{m^{2}}{x_{m n}^{2}-m^{2}} \frac{\omega^{2}}{\omega_{c m n}^{2}}\right)\right]\right\}
\end{aligned}
$$

where $\delta$ is the skin depth, and $\delta, \omega_{c m n}, r_{w}$ are all functions of $z$.

The beam current components $J_{r}$ and $J_{\theta}$ in (3) are assumed to be real quantities. In the linear regime, the beam current will have the same temporal and azimuthal dependence as the wave fields. But in the nonlinear regime, it contains all the temporal and spatial harmonics. By (2) we have neglected the beam effects on the transverse field profiles. To evaluate the axial field profile function $f(z)$, we shall treat the source term in (3) as a perturbed quantity. Under this assumption, it is only necessary to extract from $J_{\theta}$ and $J_{r}$ the harmonic component which matches that of the wave fields. Hence, we operate on both sides of (3) with

$$
\frac{\omega}{2 \pi} \int_{0}^{2 \pi / \omega} d t \int_{0}^{r_{w}} r d r \frac{1}{2 \pi} \int_{0}^{2 \pi} d \theta J_{m}\left(k_{m n} r\right) e^{i(\omega t-m \theta)}
$$

and obtain

$$
\begin{aligned}
\left(\frac{d^{2}}{d z^{2}}+k_{z}^{2}\right) f(z)= & -\frac{8 i}{x_{m n}^{2} K_{m n} \omega} \frac{1}{f^{*}(z)} \\
& \cdot \int_{0}^{r_{w}} r d r \int_{0}^{2 \pi} d \theta\left\langle\vec{J} \cdot \vec{E}^{*}\right\rangle_{t}
\end{aligned}
$$

where

$$
\begin{aligned}
\langle F\rangle_{t} & =\frac{\omega}{2 \pi} \int_{0}^{2 \pi / \omega} F d t \\
K_{m n} & =J_{m}^{2}\left(x_{m n}\right)\left(1-m^{2} / x_{m n}^{2}\right)
\end{aligned}
$$

and $\vec{E}^{*}$ is given by the complex conjugate of (2d, e). Equation (6) is coupled to the electron equations of motion through the current density $\vec{J}$ which is assumed to be a real quantity in (6).

In the case of a uniform structure, i.e., $\omega_{c m n}, \delta$ and $r_{w}$ are constants, $k_{z}^{2}$ defined by (4) or (5) becomes a constant. Hence, in the absence of the beam current, (6) yields $f(z) \propto$ $\exp \left( \pm i k_{z} z\right)$ and (2) reduces exactly to the $\mathrm{TE}_{m n}$ mode of a uniform waveguide. We verify below that (6) also conserves the energy.

From (2), we evaluate the net wave power in the positive $z$-direction as follows:

$$
\begin{aligned}
P_{\text {net }} & =\frac{c}{8 \pi} \int_{0}^{r_{w}} r d r \int_{0}^{2 \pi} d \theta \operatorname{Re}\left[\vec{E} \times \vec{B}^{*} \cdot \vec{e}_{z}\right] \\
& =\frac{i}{16} \omega x_{m n}^{2} K_{m n}\left[f(z) f^{\prime *}(z)-f^{*}(z) f^{\prime}(z)\right] .
\end{aligned}
$$

The derivative of $P_{\text {net }}$ gives

$$
\frac{d}{d z} P_{\text {net }}=\frac{i}{16} \omega x_{m n}^{2} K_{m n}\left[f(z) f^{\prime \prime *}(z)-f^{*}(z) f^{\prime \prime}(z)\right] \text {. }
$$

Substituting $f^{\prime \prime}$ from (6) into (8), we obtain

$$
\begin{aligned}
\frac{d}{d z} P_{\text {net }}= & \frac{i}{16} \omega x_{m n}^{2} K_{m n}\left(k_{z}^{2}-k_{z}^{* 2}\right)|f|^{2} \\
& -\frac{1}{2} \int_{0}^{r_{w}} r d r \int_{0}^{2 \pi} d \theta\left\langle\vec{J} \cdot\left(\vec{E}+\vec{E}^{*}\right)\right\rangle_{t} \\
= & -\frac{1}{8} \omega x_{m n}^{2} K_{m n} \frac{\omega_{m n}^{2}}{c^{2}} \frac{\delta}{r_{w}} \\
& \cdot\left(1+\frac{m^{2}}{x_{m n}^{2}-m^{2}} \frac{\omega^{2}}{\omega_{c m n}^{2}}\right)|f|^{2} \\
& -\int_{0}^{r_{w}} r d r \int_{0}^{2 \pi} d \theta\langle\vec{J} \cdot \operatorname{Re}(\vec{E})\rangle_{t}
\end{aligned}
$$

where (5) has been used to obtain the second equality in (9).

Equation (9) can be written

$$
\frac{d}{d z} P_{\text {net }}+p_{\text {ohm }}=p_{\text {in }}
$$


where $p_{\text {ohm }}$ is the Ohmic power per unit length dissipated on the wall

$$
p_{\mathrm{ohm}}=\frac{1}{8} \omega x_{m n}^{2} K_{m n} \frac{\omega_{c m n}^{2}}{c^{2}} \frac{\delta}{r_{w}}\left(1+\frac{m^{2}}{x_{m n}^{2}-m^{2}} \frac{\omega^{2}}{\omega_{c m n}^{2}}\right)|f|^{2}
$$

and $p_{\text {in }}$ is the power per unit length deposited into the RF fields by the electron beam

$$
p_{\text {in }}=-\int_{0}^{r_{w}} r d r \int_{0}^{2 \pi} d \theta\langle\vec{J} \cdot \operatorname{Re}(\vec{E})\rangle_{t} .
$$

Equation (10) is a quasi-steady-state continuity equation stating that, over any interaction length, the increase in the net wave power plus the dissipated ohmic power is equal to the power deposited into the RF fields by the electron beam, all quantities being time-averaged over one wave period.

We note that all the equations presented so far are equally valid in propagating as well as cutoff sections of a weakly nonuniform RF structure. In the case of propagating waves, the expression for the Ohmic power in (11) can be obtained directly from the local dispersion relation (5) in terms of the attenuation rate $\operatorname{Im}\left(k_{z}\right)$.

\section{B. Electron Dynamics}

We treat the electron dynamics by the standard technique [26]-[32] of orbit tracing a sufficient number of representative electrons, each governed by the relativistic equation of motion

$$
\frac{d}{d t} \vec{p}=-e \vec{E}-\frac{e}{c} \vec{v} \times\left(\vec{B}_{\text {ext }}+\vec{B}\right)
$$

where $\vec{p}=\gamma m \vec{v}, \vec{v}$ is the velocity, $\gamma=\left(1-v^{2} / c^{2}\right)^{-(1 / 2)}, \vec{E}$ and $\vec{B}$ are given by (2), and $\vec{B}_{\text {ext }}$ is the static, axisymmetric external magnetic field. $\vec{B}_{\text {ext }}$ as a function of $r$ and $z$ can be expanded into an infinite series in terms of the derivatives of $B_{\circ}(z)$, where $B_{o}(z)$ is the magnetic field on the axis. We assume that $B_{o}(z)$ varies slowly with $z$ and hence keep only the lowest order terms in the expansion,

$$
\vec{B}_{\text {ext }}(r, z) \cong-\frac{r}{2} B_{o}^{\prime}(z) \vec{e}_{r}+B_{o}(z) \vec{e}_{z}
$$

where $\vec{B}_{\text {ext }}$ satisfies $\nabla \cdot \vec{B}_{\text {ext }}=0$ exactly.

It is convenient to change the independent variable in (13) from $t$ to $z$ through the relation

$$
\frac{d}{d t}=v_{z} \frac{d}{d z}
$$

where $v_{z}$ is assumed to be positive. Hence (13) can be written

$$
v_{z} \frac{d}{d z} \vec{p}=-e \vec{E}-\frac{e}{c} \vec{v} \times\left(\vec{B}_{\mathrm{ext}}+\vec{B}\right)
$$

and $t$ is now a function of $z$ determined by

$$
\frac{d t}{d z}=\frac{1}{v_{z}} \text {. }
$$

For an electron beam represented by $N$ discrete electrons, the current density $\vec{J}$ can be written

$$
\vec{J}(\vec{x}, t)=\frac{C}{r} \sum_{j=1}^{N} W_{j} \delta\left(r-r_{j}\right) \delta\left(\theta-\theta_{j}\right) \delta\left(z-z_{j}\right) \vec{v}_{j}
$$

where $W_{j}$ is a normalized weighting factor for the $j$ th electron satisfying

$$
\sum_{j=1}^{N} W_{j}=1
$$

and $C$ is determined from the beam current $I_{b}$ through

$$
I_{b}=\int_{0}^{r_{w}} r d r \int_{0}^{2 \pi} d \theta\left\langle J_{z}\right\rangle_{t}
$$

By assumption $v_{z}$ is positive for all the electrons. Hence $z_{j}$ a single-valued function of $t$ and $\delta\left(z-z_{j}\right)$ in (18) can be converted to a function of $t$ as follows

$$
\delta\left(z-z_{j}\right)=\delta\left(t-t_{j}\right) /\left|\frac{d}{d t} z_{j}\right|_{t=t_{j}}=\delta\left(t-t_{j}\right) / v_{z j}
$$

where $t_{j}$ is the time at which the $j$ th electron reaches the position $z$ and $v_{z j}$ is its axial velocity at $z$. The $z$-component of $\vec{J}$ then becomes

$$
J_{z}(\vec{x}, t)=\frac{C}{r} \sum_{j=1}^{N} W_{j} \delta\left(r-r_{j}\right) \delta\left(\theta-\theta_{j}\right) \delta\left(t-t_{j}\right) .
$$

Substitution of (22) into (20) yields $C=2 \pi I_{b} / \omega$. Hence

$$
\vec{J}=\frac{2 \pi I_{b}}{\omega r} \sum_{j=1}^{N} W_{j} \delta\left(r-r_{j}\right) \delta\left(\theta-\theta_{j}\right) \delta\left(t-t_{j}\right) \frac{\vec{v}_{j}}{v_{z j}}
$$

where $I_{b}$ carries a negative sign.

Substituting (23) into (6), we obtain

$$
\begin{aligned}
& \left(\frac{d^{2}}{d z^{2}}+k_{z}^{2}\right) f(z) \\
& \quad=i \frac{8\left|I_{b}\right|}{x_{m n}^{2} K_{m n} \omega} \sum_{j=1}^{N} W_{j} \frac{\vec{v}_{j}(z) \cdot \vec{E}^{*}\left(r_{j}, \theta_{j}, t_{j}, z\right)}{v_{z j}(z) f^{*}(z)} .
\end{aligned}
$$

The right-hand side of (24) is a source term proportional to the beam/wave energy exchange rate averaged in time over one wave period and summed up for all the electrons. At a given position $z$, the electric field in (24) is to be evaluated at the transverse coordinates $r_{j}, \theta_{j}$ and time coordinate $t_{j}$ of the $j$ th electron at position $z \cdot r_{j}, \theta_{j}$, and $t_{j}$ are different functions of $z$ for different electrons.

\section{Initial Electron Distribution}

We now consider the representation of the initial electron beam. The quasi steady-state assumption is based on continuous beam injection. In (24), however, it is only necessary to follow the dynamics of a segment of the beam injected over the duration of one wave period since each ensuing segment will behave in an identical manner under the quasi steadystate assumption. Hence we set the initial time coordinate $t_{j}$ (at which the $j$ th electron enters the interaction region) at equally divided intervals between 0 and $2 \pi / \omega$. For the initial distribution in space, we assume that the electron beam is annular with all the guiding centers uniformly distributed on the circle of radius $r_{c}$ (no guiding center spread) and around each guiding center is a ring of electrons uniformly 


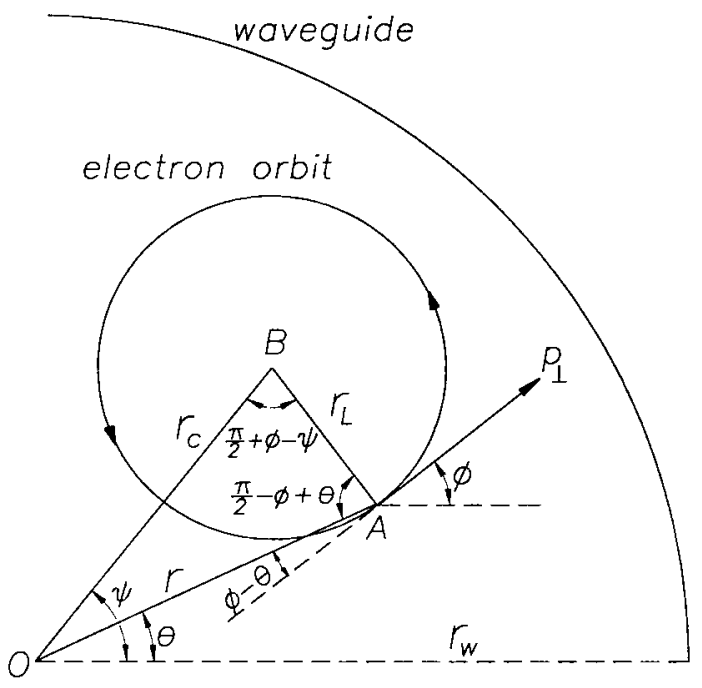

Fig. 3. Projected electron orbit (circle) based on the instantaneous position and velocity of the electron at point $\mathrm{A}$ in the presence of a uniform magnetic field. Center of the orbit (point B) defined by (A.1) and (A.2) is the instantaneous guiding center. In the absence of RF fields, point $B$ is stationary. Point $\mathrm{O}$ is the center of the waveguide.

spaced in their common gyrational orbit (see Fig. 3). For the initial momentum distribution, we assume that the electrons are monoenergetic with a pitch angle spread specified through the weighting factor $W_{j}$ by

$$
W_{j}=A \exp \left[\frac{-\left(p_{z j}-\bar{p}_{z}\right)^{2}}{2 \Delta p_{z}^{2}}\right]
$$

where $\bar{p}_{z}$ is the average axial momentum and $\Delta p_{z}$ is approximately the standard deviation from the average if $\Delta p_{z} \ll \bar{p}_{z}$. We shall equally divide the initial $p_{z j}$ between $\bar{p}_{z}-3 \Delta p_{z}$ and $\bar{p}_{z}+3 \Delta p_{z}$ where the dominant contribution comes from. The beam energy and guiding center spread, if nonnegligible, can be similarly modeled with additional electrons and appropriate modifications to the weighting factor. Sufficient number of electrons must be included in the ensemble to insure convergence.

\section{Boundary Conditions}

We assume that the interaction structure under study [Fig. 2(c)] begins and ends with a short uniform section for input-output coupling. Either the amplification or selfoscillation can be modeled through the specification of appropriate boundary conditions.

For the amplification, the boundary conditions at the left end $\left(z=z_{1}\right)$ are

$$
\begin{aligned}
f\left(z_{1}\right) & =f_{+} e^{i k_{z} z_{1}}+f_{-} e^{i k_{z} z_{1}} \quad \text { and } \\
f^{\prime}\left(z_{1}\right) & =i k_{z}\left(f_{+} e^{i k_{z} z_{1}}-f_{-} e^{-i k_{z} z_{1}}\right)
\end{aligned}
$$

where $k_{z}$ is the propagation constant in the input waveguide, $f_{+}$is given by the power and phase of the drive wave, and $f_{-}$ represents the amplitude and phase of the backward wave at $z_{1} \cdot f_{-}$is to be determined from the outgoing-wave boundary condition at the right end $\left(z=z_{2}\right)$, namely,

$$
f^{\prime}\left(z_{2}\right)=i k_{z} f\left(z_{2}\right)
$$

where $k_{z}$ is the propagation constant in the output waveguide.

In the amplifier problem, all the parameters except $f_{-}$are predetermined. Hence, $f\left(z_{2}\right)$ and $f^{\prime}\left(z_{2}\right)$, to be evaluated from (24), are both functions of $f_{-}$and we may rewrite (27) as

$$
D\left(f_{-}\right)=f^{\prime}\left(z_{2}\right)-i k_{z} f\left(z_{2}\right)=0 .
$$

The problem then becomes one of solving for the complex constant $f_{-}$as the root of the complex function $D\left(f_{-}\right)$. A root finding algorithm such as MULLER can yield $f_{-}$after a few iterations.

The field profile function $f(z)$ thus determined will be the basis for all diagnostics including the output power, gain profile, Ohmic loss profile, forward/backward wave composition, input-output phase relation, and reflections at all interfaces and discontinuities.

For the self-oscillation, we have a pure backward-wave at the left end given by the boundary conditions

$$
\begin{aligned}
f\left(z_{1}\right) & =\left|f_{-}\right| e^{-i k_{z} z_{1}} \quad \text { and } \\
f^{\prime}\left(z_{1}\right) & =-i k_{z}\left|f_{-}\right| e^{-i k_{z} z_{1}}
\end{aligned}
$$

where $\left|f_{-}\right|$is the amplitude of the backward wave at $z_{1}$. The phase of the wave is of no significance to the self-oscillation. The boundary condition at the right end is again given by (27) but the undetermined quantities are now $\left|f_{-}\right|$and the oscillation frequency $\omega$, both being real. Hence (27) can be written

$$
D\left(\left|f_{-}\right|, \omega\right)=f^{\prime}\left(z_{2}\right)-i k_{z} f\left(z_{2}\right)=0
$$

from which we solve for $\left|f_{-}\right|$and $\omega$, then proceed with the relevant diagnostics.

We have completed the set of self-contained and axially self-consistent equations for linear and nonlinear calculations. Though not necessary, it is computationally convenient and physically transparent to convert the two rapidly varying position coordinates $r$ and $\theta$ of the electron into slowly varying position coordinates $r_{c}$ and $\psi$ of the instantaneous guiding center (Fig. 3) and rewrite all equations in slow variables. This can be done by straightforward algebraic manipulations. The results are given in the Appendix.

\section{A High-Gain SchEmE AND Stability AnAlysis}

We shall show that the distributed-loss structure of Fig. 2(c) can yield ultra high stable gain. The scheme is based on the different responses to wall losses between the cold tube and hot tube modes. The cold tube mode has all of its energy in the electromagnetic fields. In a hot tube, however, energy of the beam generated mode resides not only in the electromagnetic fields but also in the kinetic energy of the oscillatory motion of the electrons, the latter being an integral part of the hot tube mode. The lossy wall absorbs the electromagnetic energy but not the oscillatory energy of the electrons. Thus, wall losses of the amplifier circuit attenuate the reflected wave (basically a cold tube mode) significantly more than they reduce the gain of the amplifying wave (a hot tube mode). It can be shown analytically [33], that the reduction in hot tube gain due to wall losses is only one third of the cold tube attenuation over the same distance. 
Such unequal effects can be exploited simultaneously to achieve both high gain and stability. In Fig. 2(c), the lossy and conducting wall sections comprise the basic linear and nonlinear stages of the amplification, respectively. The linear section is made sufficiently long to provide the desired gain, while the nonlinear section is constrained to a minimum length to enhance the threshold of absolute instabilities. As in previous experiments [6], [8], [13], we amplify the $\mathrm{TE}_{11}$ waveguide mode in a uniform magnetic field by the fundamental cyclotron harmonic interaction (Fig. 1, point 3).

We first analyze three types of oscillations associated with the configuration of Fig. 2(c) using a code based on the formalism of Section II. Incorporation of the loss factor in (5) makes it possible to follow the beam and wave dynamics throughout the entire structure. On the other hand, imposition of physical boundary conditions at both ends allows the evaluation of a self-consistent RF field profile, $f(z)$, to account for wave reflections at the lossy wall interfaces and structural nonuniformities. Such details in the modeling allow oscillations of various origins to be studied on the basis of the overall rather than sectionized interaction structure.

Properties of oscillations are illustrated in Figs. 4-8 for the structural dimensions of [13] shown in Fig. 4(a), (b). However, the qualitative features are of a general nature. In Figs. 4-8, a cold beam $\left(\Delta v_{z} / v_{z}=0\right)$ has been assumed for the analysis of oscillations. This is a good approximation since the oscillations, generally occurring near the cut-off frequency, are relatively insensitive to the beam velocity spread. In subsequent calculations (Section IV) for comparison with the measured power and gain, inclusion of a velocity spread becomes essential. Also in some cases, the beam current, magnetic field, and wall resistivity are chosen to be different from those in actual conditions in order to bring out the oscillation (as in Fig. 4) or to show the transition from the stable regime to the regime of spurious oscillation (as in Figs. 5-8).

\section{A. Global Reflective Oscillations}

Reflective oscillation starts when the total gain exceeds the reflection at the input-output ends plus the attenuation in the lossy section (all in $\mathrm{dB}$ ). The higher the reflections at either end, the lower the start-oscillation current. For the configuration under study [Figs. 2(c) or 4(a), (b)], reflections take place in the input-output tapers and the coupling holes at $z_{1}$ and $z_{2}$. Reflections from the tapers are implicit in the formalism, but reflections from the coupling holes are artificially modeled by a discontinuity in wall radius at $z_{1}$ and $z_{2}$.

Fig. 4(c) shows the field profile of a typical global reflective oscillation. The field profile is essentially that of an amplified wave where the ripples are caused by beating with the reflected wave. As a noticeable feature, the field extends from end to end over the entire structure including the input section. Fig. 5 shows the reflective oscillation power (coming out of the right end) as functions of beam current $I_{b}$, lossy section length $L_{1}$, wall resistivity $\rho$ of the lossy section, and magnetic field $B_{o}$. In Fig. 5, $\rho$ is normalized to that of copper $\left(\rho_{\mathrm{cu}}=\right.$

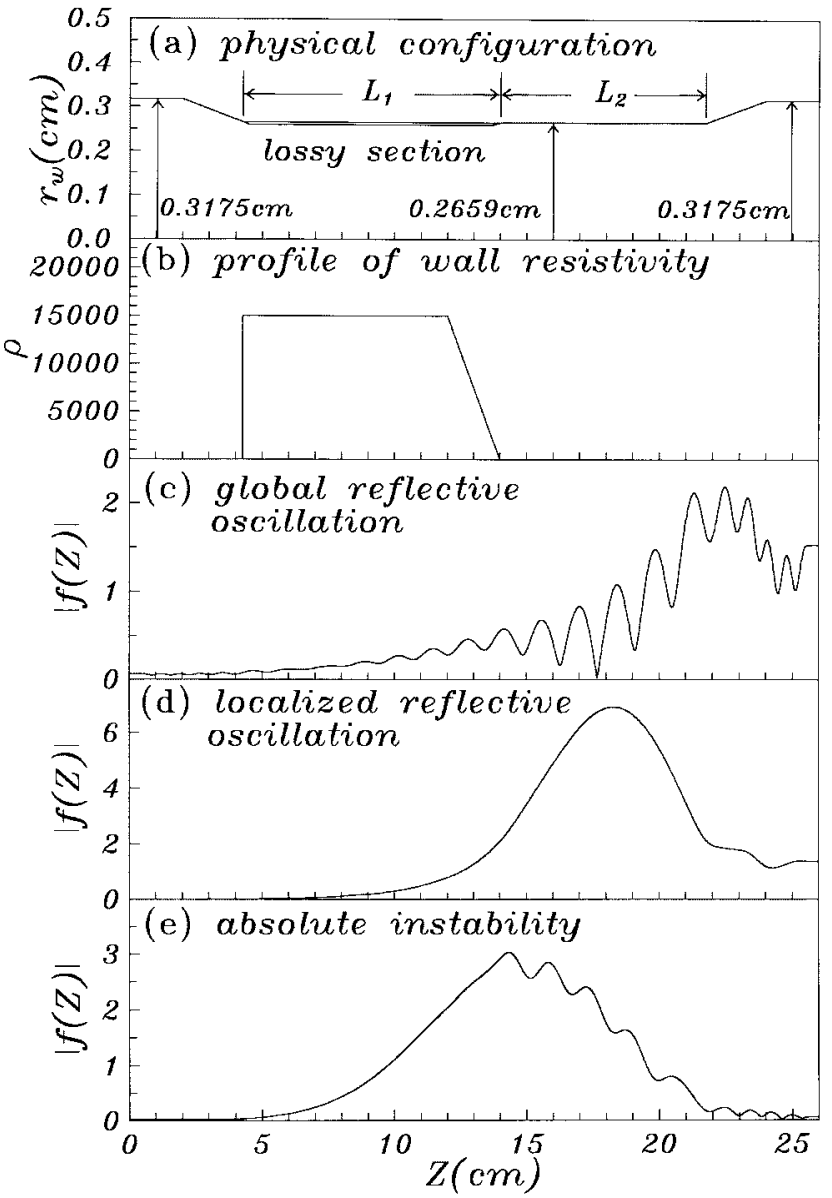

Fig. 4. Calculated profiles of the RF field amplitude $|f(z)|$ in the distributed-loss structure of [13] shown in (a) and (b), for (c) the global reflective oscillation, (d) the localized reflective oscillation, and (e) the absolute instability.

$\left.1.72 \times 10^{-8} \Omega \mathrm{m}\right)$. As shown, the oscillation can be stabilized by lowering the operating current [Fig. 5(a)] and magnetic field [Fig. 5(d)], both having the effect of reducing the gain, or by increasing the wall resistivity [Fig. 5(c)] to reduce the feedback. It is interesting to note that a longer lossy section not only increases the gain (not shown in Fig. 5) but also results in zero-drive stability [Fig. 5(b)]. Beam current is usually fixed by the power requirement and the magnetic field must be fine tuned for maximum efficiency, hence wall resistivity provides the most effective means for stabilization. Wall resistivity reduces the gain, but this can be easily compensated by a lengthened lossy section.

\section{B. Localized Reflective Oscillations}

The conducting-wall section [of length $L_{2}$ in Fig. 4(a)] by itself is subject to localized oscillations due to reflections at the lossy-wall junction on the left and the output structure on the right. Fig. 4(d) illustrates the field profile of such an oscillation in the $\mathrm{TE}_{111}$ mode. It is seen that the field is localized to the conducting-wall section. Since it is the lowest order axial mode $(\ell=1)$, its wavelength is much longer than that of the global oscillation mode [cf., Fig. 4(c)] which occurs in the high gain region of the growth spectrum and hence at 

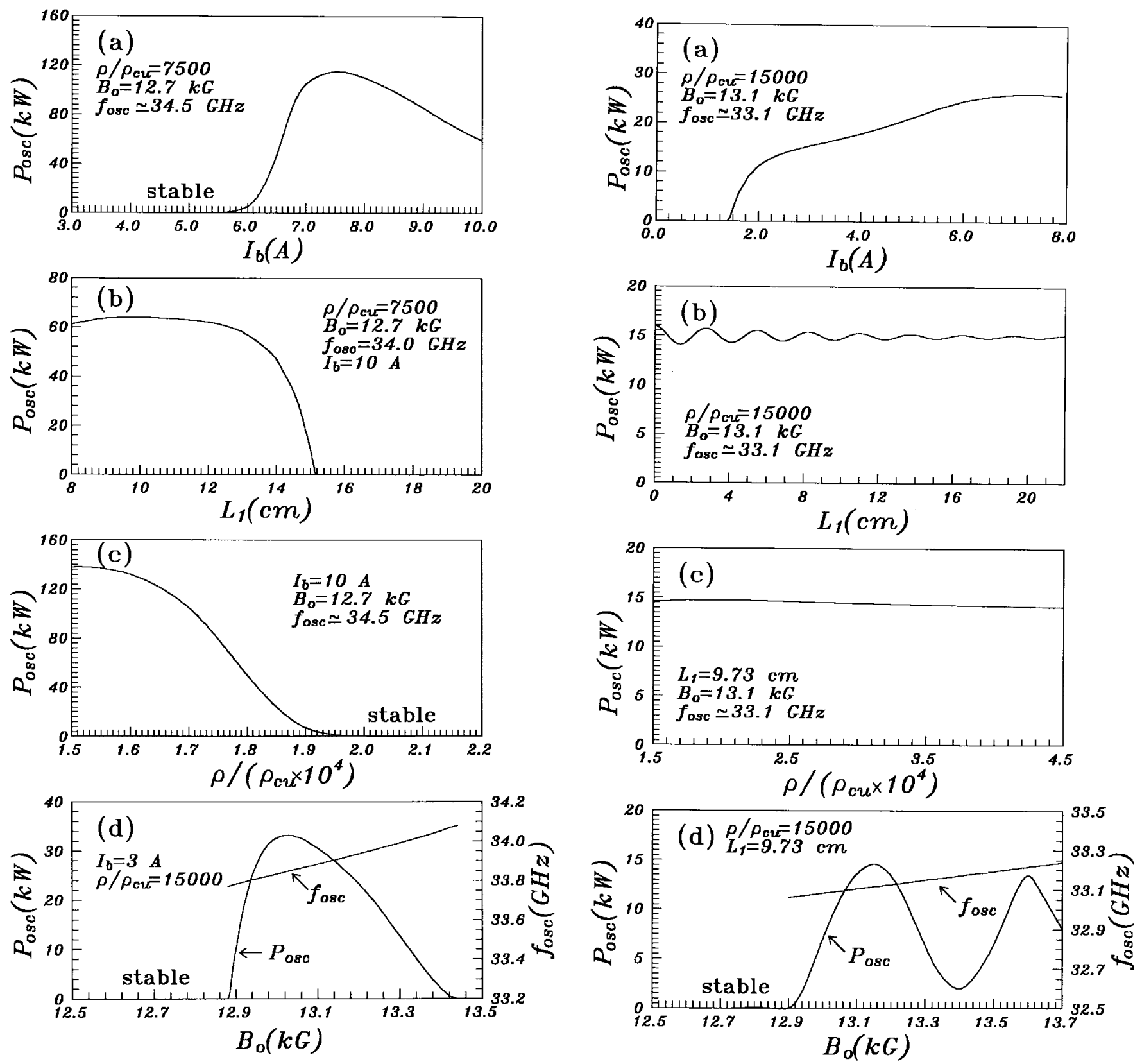

Fig. 5. Calculated power of global reflective oscillations ( $\mathrm{TE}_{11}$ mode, $\left.s=1\right)$ versus (a) beam current $I_{b}$, (b) length $L_{1}$ of the lossy section, (c) wall resistivity (of the lossy section), and (d) magnetic field $B_{o}$. Parameters used are [refer to Fig. 2(c)] $L_{1}=9.73 \mathrm{~cm}, L_{2}=7.73 \mathrm{~cm}, V_{b}=100 \mathrm{kV}$, $\alpha=0.85, r_{c}=0.09 \mathrm{~cm}$, and $\Delta V_{z} / V_{z}=0$. Wall resistivity of the lossy section is normalized to that of copper $\left(\rho_{\mathrm{cu}}=1.72 \times 10^{-8} \Omega \mathrm{m}\right)$.

a higher frequency. Fig. 6 shows the characteristics of such oscillation in the lowest order $\left(\mathrm{TE}_{111}\right)$ axial mode. As in the global reflective oscillation, lower beam current always results in stability [Fig. 6(a)]. But since the oscillation is localized to the conducting-wall section, the oscillation power (out of the right end) is almost independent of the length $\left(L_{1}\right)$ and wall resistivity $(\rho)$ of the lossy section [Fig. 6(b) and (c)]. These two features are qualitatively different from those of the global reflective oscillation which is highly sensitive to $L_{1}$ and $\rho$. Because it cannot be effectively suppressed by wall losses, the localized reflective oscillation has been observed

Fig. 6. Calculated power of localized reflective oscillations $\left(\mathrm{TE}_{111}\right.$ mode, $s=1$ ) in the conducting-wall section versus (a) beam current $I_{b}$, (b) length $L_{1}$ of the lossy section, (c) wall resistivity $\rho$ of the lossy section, and (d) magnetic field $B_{o} . I_{b}=3 \mathrm{~A}$ and other parameters are the same as in Fig. 5 unless denoted otherwise. The oscillation cannot be stabilized by varying the length or wall resistivity of the lossy section.

in our experiment (Section IV), but in a range of magnetic field higher than the optimum magnetic field for the amplified mode. Hence such oscillations could be avoided in the normal operating magnetic field range of the gyro-TWT.

The frequency and power of the localized oscillation as functions of the magnetic field are shown in Fig. 6(d). For both the global and localized reflective oscillations, the magnetic field exerts a frequency pulling effect (Figs. 5(d) and 6(d)]. It is interesting to note that such effect is approximately 5 times stronger for the global oscillation than for the localized oscillation. The reason is that localized oscillation occurs in 


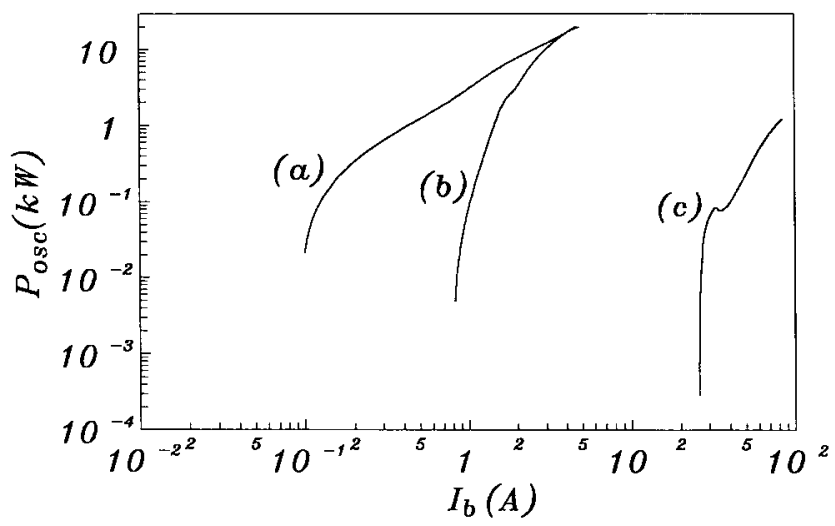

Fig. 7. Calculated power of the second harmonic absolute instability $\left(\mathrm{TE}_{21}\right.$ mode, $f_{\text {osc }} \cong 56 \mathrm{GHz}$ ) versus the beam current for (a) the unsevered, (b) the severed, and (c) the distributed-loss structures of Fig. 2, all having a total interaction length of $17.46 \mathrm{~cm} . V_{b}=100 \mathrm{kV}, \alpha=0.85, r_{c}=0.09 \mathrm{~cm}$, $\Delta V_{z} / V_{z}=0$, and $B_{o}=12.54 \mathrm{kG}$. In (b), the $3 \mathrm{~cm}$ sever has a cold circuit loss of $\sim 30 \mathrm{~dB}$. In (c), the $9.73 \mathrm{~cm}$ lossy section has a cold circuit loss of $\sim 20 \mathrm{~dB}$. Start-oscillation currents for cases (a), (b), and (c) are, respectively, $0.1,0.9$, and $26 \mathrm{~A}$.

a structure much like a gyromonotron resonator. Boundary effects make the resonant mode more resistant to frequency pulling.

\section{Absolute Instability}

In our experiments (Section IV and [6], [8], and [13]), the $\mathrm{TE}_{21}$ mode oscillation at the second cyclotron harmonic (Fig. 1, point 2) was the only absolute instability observed. Fig. 4(e) illustrates the field profile of such an oscillation in the distributed-loss interaction structure. The field profile and power flow (in the negative $z$-direction) are characteristic of a backward wave. The lossy section, located at the field maximum and in the direction of the power flow, causes strong damping of the mode, resulting in a much higher start-oscillation current. Fig. 7, taken from [13], shows the calculated power (coming out of the left end) of the $\mathrm{TE}_{21}$ mode, second harmonic oscillation as a function of the beam current for the unsevered [Fig. 2(a)], severed [Fig. 2(b)], and distributed-loss (Figs. 2(c), or 4(a), (b)] interaction circuits of identical total length and operating parameters. The start-oscillation current shows a dramatic increase with the distributed-loss structure for reasons just discussed.

Fig. 8 displays the start-oscillation current $\left(I_{s t}\right)$ of the same mode in a broader parameter space for the distributed-loss structure. Fig. 8(a) plots $I_{s t}$ versus the electron pitch angle $\alpha\left(=v_{\perp} / v_{z}\right)$ for different values of $\rho . I_{s t}$ decreases with increasing $\alpha$ as expected. Again, the wall resistivity is shown to be highly effective in stabilizing the absolute instability. In the experiment presented in the following section, we increased the wall resistivity and were able to operate stably at higher $I_{b}$ and $\alpha$ values to achieve higher power and efficiency.

Fig. 8(b) shows the dependence of $I_{s t}$ on the length $\left(L_{1}\right)$ of the lossy section for different amount of wall losses. Increasing $L_{1}$ has the effect of lowering $I_{s t}$. This is because the backward wave, while being damped in the lossy section, still interacts with the beam. Although fields of the absolute instability

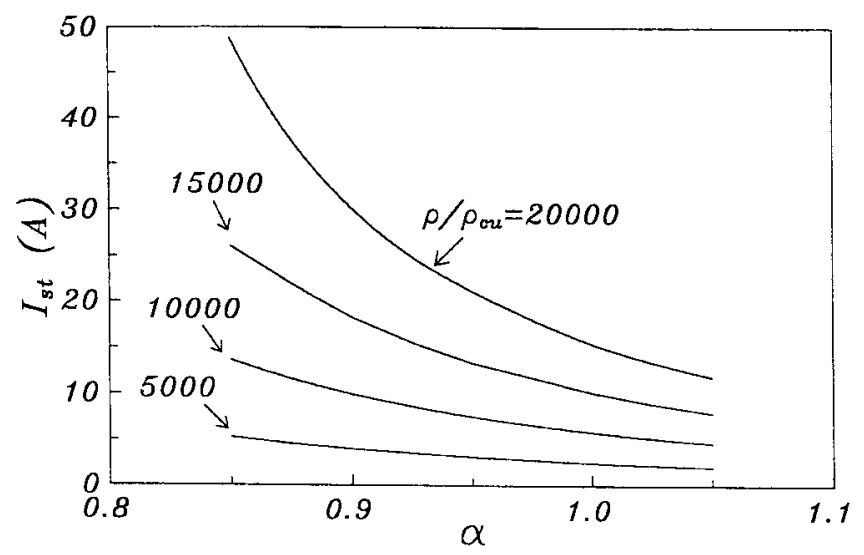

(a)

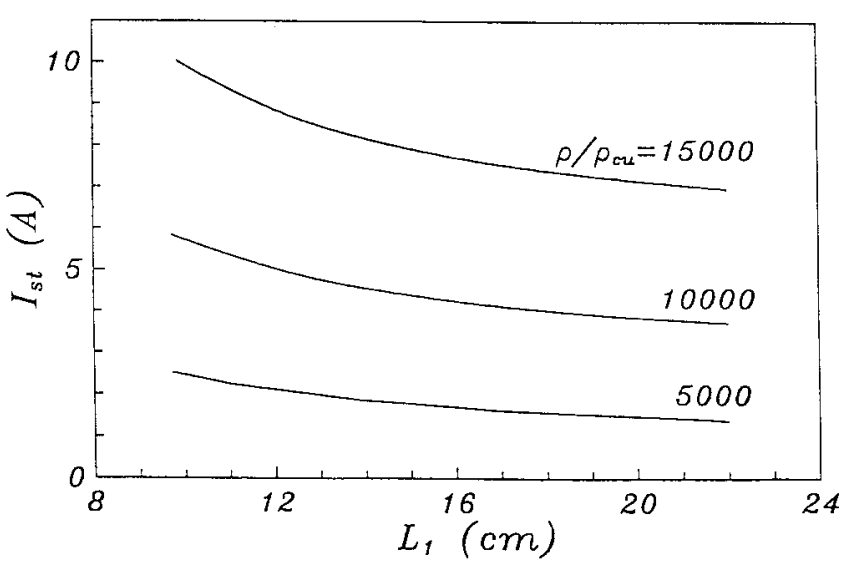

(b)

Fig. 8. Calculated start-oscillation current of the second harmonic absolute

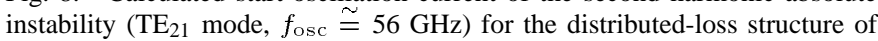
Fig. 2(c) versus (a) electron pitch angle $\alpha$ and (b) length $L_{1}$ of the lossy section. In both (a) and (b), $L_{2}=7.73 \mathrm{~cm}, V_{b}=100 \mathrm{kV}, r_{c}=0.09 \mathrm{~cm}$, $\Delta V_{z} / V_{z}=0$, and $B_{o}=12.54 \mathrm{kG}$. In (a) $L_{1}=9.73 \mathrm{~cm}$ and in (b) $\alpha=1$.

concentrate in the conducting-wall section, weak interaction in the lossy section slightly elongates the effective interaction length to cause the reduction in $I_{s t}$. This effect is not so significant and can be compensated by increased wall losses.

\section{AN EXPERIMENTAL GYRO-TWT WITH ULTRAHIGH GAIN}

The experimental setup is similar to that of [13]. Fig. 9 shows a photo of the gyro-TWT as detached from the superconducting magnet. A mechanically tunable magnetron injection electron gun [34] is attached to the structure of Fig. 2(c). Length $L_{1}$ of the graphite-coated lossy section is increased from $9.73 \mathrm{~cm}$ (of [13]) to $20 \mathrm{~cm}$ with a much higher cold circuit loss of $\sim 100 \mathrm{~dB}$ (corresponding to $\rho=$ $3.6 \times 10^{4} \rho_{\mathrm{cu}}$ ), while length $L_{2}$ of the conducting-wall section is shortened from $7.23 \mathrm{~cm}$ to $4 \mathrm{~cm}$. These changes have been made in order to achieve high gain as well as overall stability. To insure stability under the worst input-output matching conditions, the wall resistivity employed in the experiment is considerably higher than the theoretically required minimum. The graphite solution (DAG 154) was coated with a hand held brush on the inner wall of waveguide to form a resistive layer. The desired loss was achieved experimentally by the thickness 


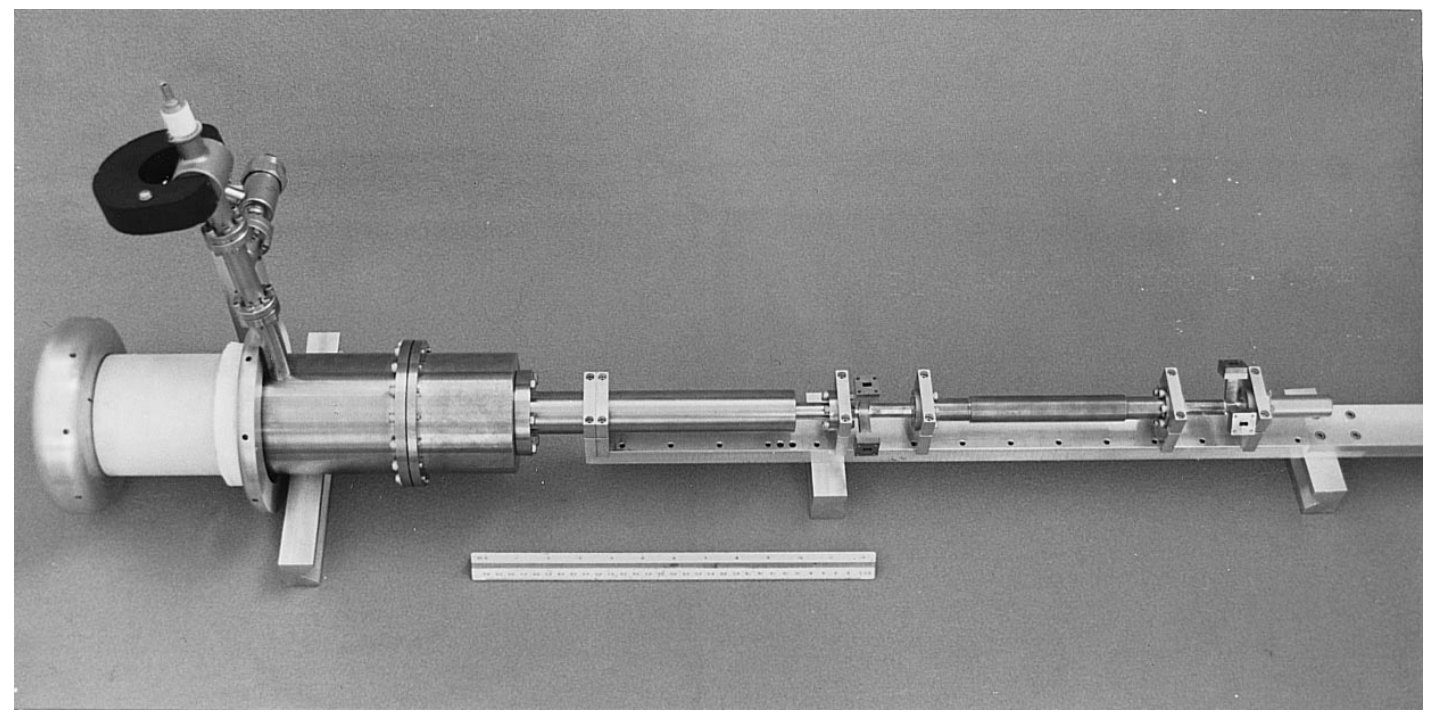

Fig. 9. Photo of the Ka-band gyro-TWT detached from the superconducting magnet.

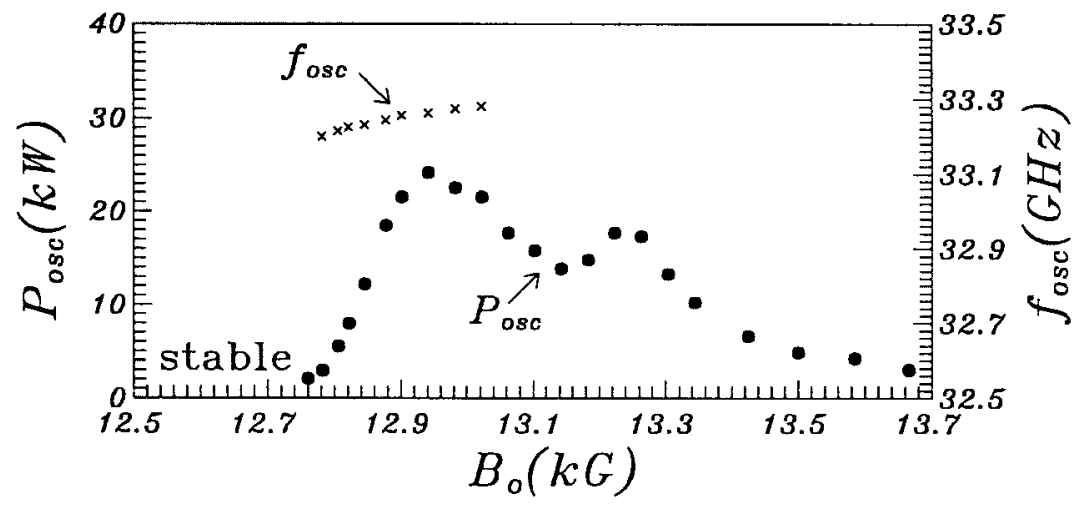

Fig. 10. Measured power (dots) and frequency (crosses) of spurious oscillations at zero drive power versus the magnetic field. Optimum operating magnetic field (for Figs. 11 and 13) of the gyro-TWT lies in the stable region. $L_{1}=20 \mathrm{~cm}, L_{2}=4 \mathrm{~cm}, V_{b}=100 \mathrm{kV}$ and $I_{b}=3.5 \mathrm{~A}$.

of the layer which, when dried, is approximately equal to the skin depth $(\sim 0.007 \mathrm{~cm})$. The coating process does not insure uniformity of the layer. However, mode conversion is not expected of the lowest order $\left(\mathrm{TE}_{11}\right)$ mode being amplified.

Input-output waves are coupled at $z_{1}$ and $z_{2}$ through the side walls with newly designed dual-port couplers [i.e., the two end sections connected to the uniform interaction section of Fig. 2(c)]. By slightly squeezing its cross-section, each coupler also functions as a converter between circularly and linearly polarized waves [6], [35]. The dual-port couplers provide an additional benefit of critical importance for studies of oscillations. The couplers are designed so that the drive wave enters through one input port and the amplified $\mathrm{TE}_{11}$ circularly polarized wave exits through only one output port (with $\sim 30 \mathrm{~dB}$ isolation from the other output port), while the non- $\mathrm{TE}_{11}$ modes exit equally from both ports at either the input or output end. This allows even very low-level oscillations of non- $\mathrm{TE}_{11}$ modes to be observed at the extra input and output ports without interference from a large-amplitude $\mathrm{TE}_{11}$ wave. The magnetic field (uniform in the interaction region) is provided by a superconducting magnet system. Output power is measured with a calibrated crystal detector (with estimated accuracy of $\pm 5 \%$ ) and verified with a calorimeter (agreement was within $\sim 5 \%$ ).

Beam parameters for the data presented in Figs. 10-13 are $V_{b}=100 \mathrm{kV}$ and $I_{b}=3.5 \mathrm{~A}$. The simulated beam velocity spread $\left(\Delta v_{z} / v_{z}\right)$ is $\sim 5 \%$ with a pitch angle $(\alpha)$ tuning range of $0.8-1.1$ [34]. To optimize the gyro-TWT performance, the pitch angle can be varied by trim coil current tuning and mechanical adjustment of the center electrode position [34]. The simulated electron guiding center position is $r_{c}=0.09 \mathrm{~cm}$.

Stability properties of the gyro-TWT at zero drive power are shown in Fig. 10. The gyro-TWT was found to be stable from all three types of instabilities considered in Section III in the optimum range of the operating magnetic field (12.65 $\left.\mathrm{kG}<B_{\circ}<12.7 \mathrm{kG}\right)$ As the magnetic field was increased, a localized reflective oscillation was observed and identified to be the $\mathrm{TE}_{111}$ mode of the conducting-wall section. As the magnetic field was increased further, the oscillation frequency became unmeasurable due to mode jumping. In Fig. 10, characteristics of the observed oscillation power and frequency as functions of the magnetic field are very similar to the theoretically predicted behavior shown in Fig. 6(d). 
TABLE II

Comparison of the Experimental Gyro-TWT with the State-OF-the-Art TWT (Varian/CPI Model VTA 5701)

\begin{tabular}{cccccccc}
\hline type & voltage & current & magnet & peak power & efficiency & gain & bandwidth \\
\hline gyro-TWT & $100 \mathrm{kV}$ & $3.5 \mathrm{~A}$ & cryogenic & $93 \mathrm{~kW}$ & $26.5 \%$ & $70 \mathrm{~dB}$ & $8.6 \%$ \\
TWT & $45 \mathrm{kV}$ & $7 \mathrm{~A}$ & solenoid & $50 \mathrm{~kW}$ & $16 \%$ & $40 \mathrm{~dB}$ & $6 \%$ \\
\hline
\end{tabular}

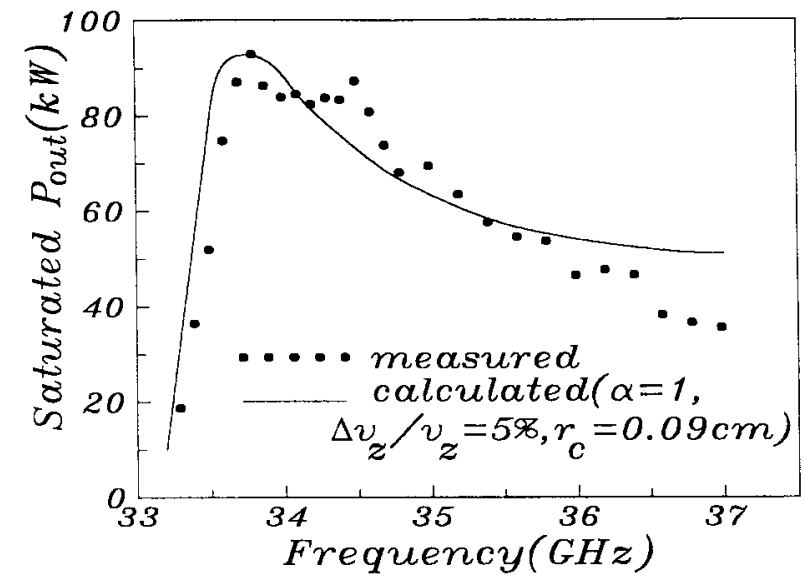

(a)

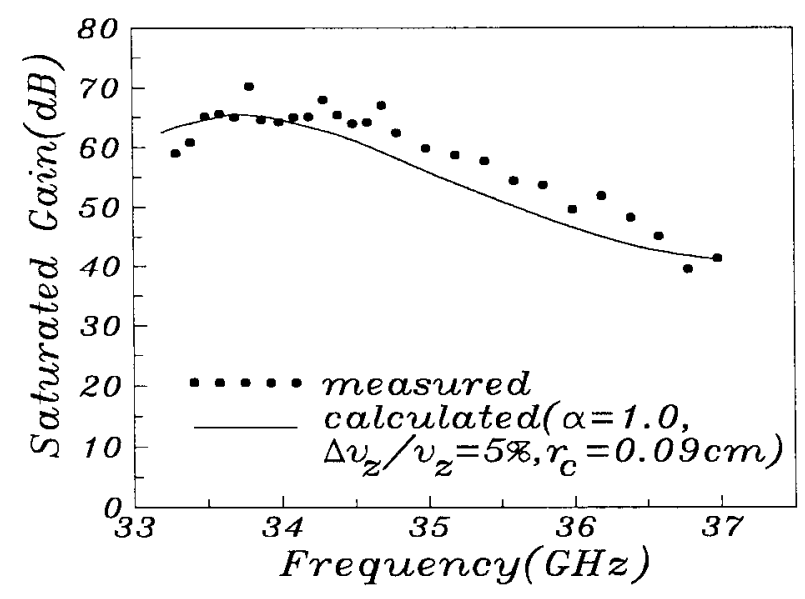

(b)

Fig. 11. Saturated output power and gain versus the frequency. Measured and calculated data are shown by dots and lines, respectively. Measured parameters (also used in the calculation) are $L_{1}=20 \mathrm{~cm}, L_{2}=4 \mathrm{~cm}$, $V_{b}=100 \mathrm{kV}, I_{b}=3.5 \mathrm{~A}$ and $B_{o}=12.7 \mathrm{kG}$. The calculation assumes $\alpha=1, \Delta V_{z} / V_{z}=5 \%$, and $r_{c}=0.09 \mathrm{~cm}$. Resistivity of the lossy section used in the calculation $\left(\rho=3.6 \times 10^{4} \rho_{\mathrm{cu}}\right)$ corresponds to the measured clod circuit loss of $\sim 100 \mathrm{~dB}$ near the cutoff frequency.

Fig. 11 plots the saturated output power and gain (dots) from the measurements as functions of the frequency. The peak power of $93 \mathrm{~kW}$ corresponds to a saturated gain of $70 \mathrm{~dB}$ at $26.5 \%$ interaction efficiency. Saturated output power over $80 \mathrm{~kW}$ with a gain above $65 \mathrm{~dB}$ was observed over a bandwidth of $1 \mathrm{GHz}$. The full-width half-maximum bandwidth is $3 \mathrm{GHz}$, approximately $8.6 \%$ of the center frequency. The ultra high gain, $30 \mathrm{~dB}$ beyond that previously achieved (Table I), permits the use of solid state sources as drivers. Fig. 12 shows typical traces of the voltage, current, drive power, and saturated output power.

Calculations have been performed for the same set of experimental parameters and assuming $\alpha=1, r_{c}=0.09 \mathrm{~cm}$, and $\left(\Delta v_{z} / v_{z}\right)=5 \%$. The saturated output power and gain are

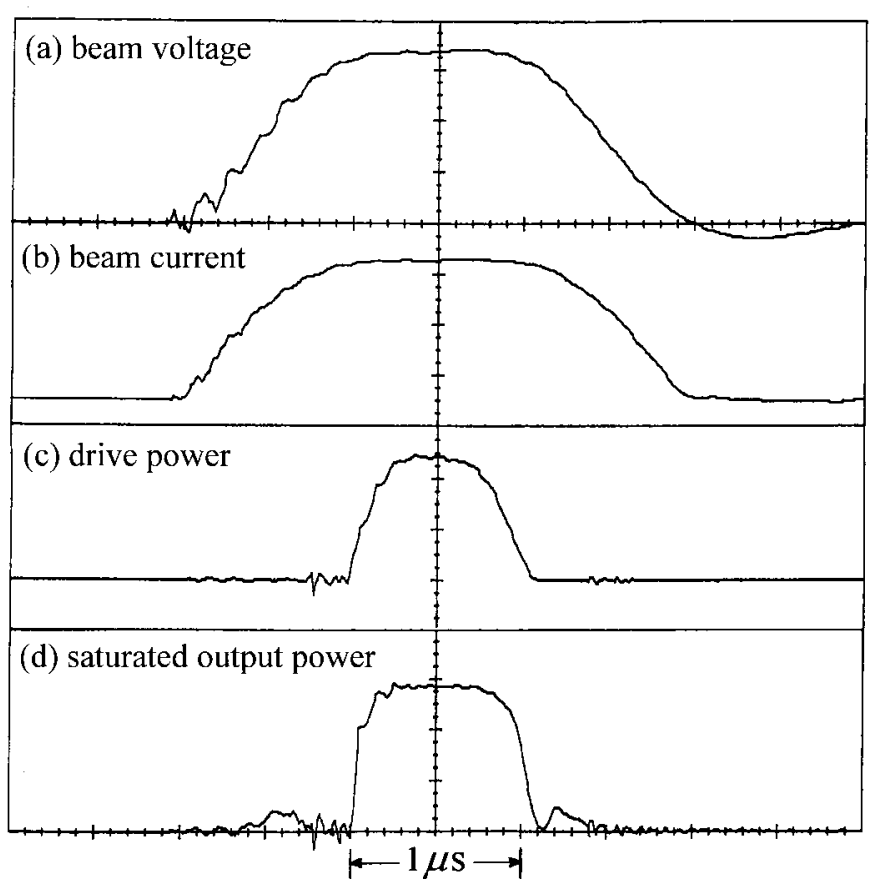

Fig. 12. Typical traces of (a) beam voltage, (b) beam current, (c) drive power, and (d) saturated output power.

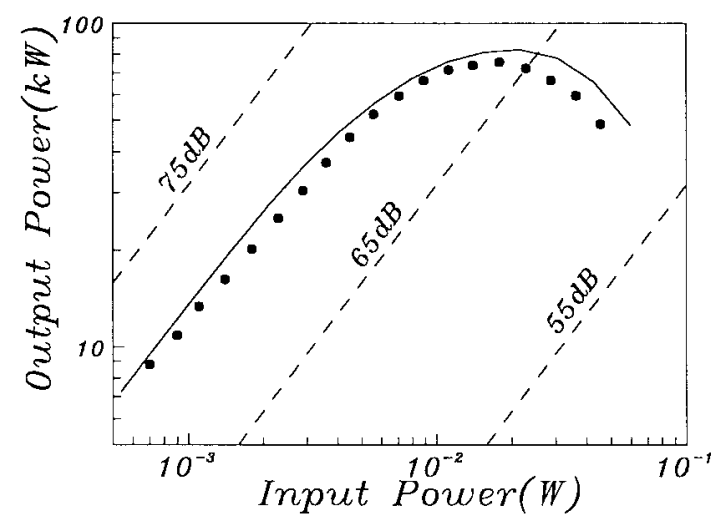

Fig. 13. Measured (dots) and calculated (line) output power versus the drive power. Drive frequency is $34.2 \mathrm{GHz}$. Other parameters are the same as Fig. 11.

plotted in Fig. 11 (solid lines) for comparison with measured data. Measured data are closely matched by theoretical predictions. Improved stability has permitted operation at a higher $\alpha$ value $(\alpha \simeq 1$ ) than that of [13] (where $\alpha \simeq 0.85$ ). This has been a contributing factor to the higher efficiency observed (26.5\% here versus $21 \%$ in [13]). The theory also predicts that the saturated power is almost independent of the length of the lossy section $\left(L_{1}\right)$ while the gain increases linearly with $L_{1}$.

Fig. 13 shows the measured output power versus the input power (dots). Linear and saturated behaviors are consistent with the calculated data (solid line). Again, $\alpha=1, \Delta v_{z} / v_{z}=$ $5 \%$, and $r_{c}=0.09 \mathrm{~cm}$ were assumed in the calculations. 
In all the measurements for Figs. 11 and 13, we have not detected any spurious oscillation. Table II compares the key parameters of the proof-of-principle gyro-TWT with those of the state-of-the-art TWT.

These studies indicate that a basic understanding of the intricate interplay between the absolute/convective instabilities, circuit losses, and reflective feedback is of fundamental importance to the scientific demonstration of the potential capability of the gyro-TWT. Significantly higher power can be obtained by employing the harmonic cyclotron maser interaction [14], [25].

Of equal importance are a variety of technological issues. For examples, better matched input-output couplers will be effective in suppressing the reflective oscillations. For the gyro-TWT under study, all types of oscillation in the interaction section have been suppressed by the wall losses. However, oscillations inside the couplers (which start at a beam current slightly above $3.5 \mathrm{~A}$ ) have limited our maximum stable operating beam current to $3.5 \mathrm{~A}$. Shorter couplers are expected to alleviate this problem and significantly increase the beam current and power. In addition, wall losses will be limiting factor in high average power operation. For the case of Fig. 13, the peak Ohmic power dissipated on the lossy walls is calculated to be approximately $5 \mathrm{~kW}$ at saturation ( $\sim 6 \%$ of the output power). For uniform resistivity in the lossy section, this results in $\sim 900 \mathrm{~W} / \mathrm{cm}$ heat dissipation at the field maximum. If the wall resistivity is profiled to evenly distributed the heat, the heat dissipation will be $\sim 150 \mathrm{~W} / \mathrm{cm}$ throughout the lossy section. The average-power handling capability will thus be limited by the availability of proper heat-resistant lossy materials and advanced cooling techniques. Supplementary attenuation by broadband side-wall coupling to an external load could conceivably be implemented to remove this limitation.

\section{APPENDIX}

\section{CONVERSION TO SLOW VARIABLES}

The electron position coordinates $r$ and $\theta$ are rapidly oscillating functions of $z$. For convenience and clarity, we may define the position coordinates $r_{c}$ and $\psi$ of the instantaneous guiding center (Fig. 3, point B) in terms of $r, \theta$, and perpendicular momentum $\vec{p}_{\perp}$ of the electron by the following equations (refer to triangle $\mathrm{OAB}$ in Fig. 3):

$$
\begin{aligned}
& r \sin \left(\frac{\pi}{2}-\varphi+\theta\right)=r_{c} \sin \left(\frac{\pi}{2}+\varphi-\psi\right) \\
& r \cos \left(\frac{\pi}{2}-\varphi+\theta\right)+r_{c} \cos \left(\frac{\pi}{2}+\varphi-\psi\right)=r_{L}
\end{aligned}
$$

where $\varphi$ is the polar angle of $\vec{p}_{\perp}$ and $r_{L}=p_{\perp} /\left(m_{e} \Omega_{e}\right)$ with $\Omega_{e}=e B_{o} / m_{e} c$.

The field functions can be expressed in terms of the slowly varying variables $r_{c}$ and $\psi$ by the Bessel function summation theorem (Fig. 3, triangle OAB)

$$
\begin{aligned}
& J_{m}\left(k_{m n} r\right) e^{-i m((\pi / 2)-\varphi+\theta)} \\
& \quad=\sum_{q=-\infty}^{\infty} J_{m+q}\left(k_{m n} r_{L}\right) J_{q}\left(k_{m n} r_{c}\right) e^{-i q((\pi / 2)+\varphi-\psi)}
\end{aligned}
$$

Substituting (2d) and (2e) for $\vec{E}^{*}$ into (24) and employing (A.3) and the Bessel function recursion formulas, we obtain

$$
\begin{aligned}
\left(\frac{d^{2}}{d z^{2}}+k_{z}^{2}\right) f(z)= & \frac{-8 k_{m n}\left|I_{b}\right|}{x_{m n}^{2} K_{m n} c} \sum_{j=1}^{N} \sum_{s=-\infty}^{\infty} W_{j} \frac{p_{\perp j}}{p_{z j}} \\
& \cdot J_{s-m}\left(k_{m n} r_{c j}\right) J_{s}^{\prime}\left(k_{m n} r_{L j}\right) e^{i A_{s j}}
\end{aligned}
$$

where

$$
A_{s j}=\omega t_{j}-s \varphi_{j}+(s-m) \psi_{j}+\left(m-\frac{s}{2}\right) \pi .
$$

The wave equation in the form of (A.4) has the clear advantage of a source term analytically separated into cyclotron harmonic components. The factor $J_{s-m}\left(k_{m n} r_{c j}\right) J_{s}^{\prime}\left(k_{m n} r_{L j}\right)$ on the right hand side indicates the strength of beam/wave coupling at the sth cyclotron harmonic.

For the equation of motion, we rewrite (A.1) and (A.2) as

$$
\begin{aligned}
r e^{-i(\varphi-\theta)} & =-i r_{L}+r_{c} e^{-i(\varphi-\psi)} \quad \text { or } \\
r_{c} e^{i \psi} & =r e^{i \theta}+i r_{L} e^{i \varphi} .
\end{aligned}
$$

Replacing $r_{L}$ with $p_{\perp} / m_{e} \Omega_{e}$ and differentiating (A.6) with respect to $z$

$$
\frac{d}{d z}\left(r_{c} e^{i \psi}\right)=\frac{d}{d z}\left(r e^{i \theta}\right)+i \frac{d}{d z}\left(\frac{p_{\perp}}{m_{e} \Omega_{e}} e^{i \psi}\right)
$$

The first term on the right hand-side of (A.7) can be written

$$
\frac{d}{d z}\left(r e^{i \theta)}=\frac{1}{v_{z}} \frac{d}{d t}(x+i y)=\frac{\gamma m_{e}}{p_{z}}\left(v_{x}+i v_{y}\right)=\frac{p_{\perp}}{p_{z}} e^{i \varphi} .\right.
$$

Substituting (A.8) into (A.7) and equating the real and imaginary parts, we obtain

$$
\begin{aligned}
\frac{d r_{c}}{d z}= & \frac{1}{m_{e} \Omega_{e}}\left[p_{\perp}\left(\frac{m_{e} \Omega_{e}}{p_{z}}-\frac{d \varphi}{d z}\right) \cos (\varphi-\psi)\right. \\
& \left.-\left(\frac{d p_{\perp}}{d z}-p_{\perp} \frac{\Omega_{e}^{\prime}}{\Omega_{e}}\right) \sin (\varphi-\psi)\right]
\end{aligned}
$$

and

$$
\begin{aligned}
\frac{d \psi}{d z}= & \frac{1}{r_{c} m_{e} \Omega_{e}}\left[\left(\frac{d p_{\perp}}{d z}-p_{\perp} \frac{\Omega_{e}^{\prime}}{\Omega_{e}}\right) \cos (\varphi-\psi)\right. \\
& \left.+p_{\perp}\left(\frac{m_{e} \Omega_{e}}{p_{z}}-\frac{d \varphi}{d z}\right) \sin (\varphi-\psi)\right]
\end{aligned}
$$

where $\Omega_{e}^{\prime}=d \Omega_{e} / d z$.

Substituting (2) and (14) for the fields in (16) and applying the Bessel function summation theorem and recursion formulas, we obtain from (16), (17), (A.9), and (A.10) the equations of motion for a single electron

$$
\begin{aligned}
\frac{d r_{c}}{d z}= & \frac{k_{m n} e}{p_{z} c} \operatorname{Re} \sum_{s=-\infty}^{\infty} i\left\{-\frac{\gamma}{\Omega_{e}}\left[\omega f(z)+\frac{i p_{z}}{\gamma m_{e}} f^{\prime}(z)\right]\right. \\
& \cdot J_{s-m}^{\prime}\left(k_{m n} r_{c}\right) J_{s}\left(k_{m n} r_{L}\right) e^{-i A_{s}} \\
& +\frac{k_{m n} r_{L}}{2} f(z)\left[J_{s-1-m}\left(k_{m n} r_{c}\right) J_{s-1}\left(k_{m n} r_{L}\right)\right. \\
& \left.\left.-J_{s+1-m}\left(k_{m n} r_{c}\right) J_{s+1}\left(k_{m n} r_{L}\right)\right] e^{-i A_{s}}\right\} \\
& -\frac{1}{2} \frac{\Omega_{e}^{\prime}}{\Omega_{e}}\left[r_{c}-r_{L} \sin (\varphi-\psi)\right]
\end{aligned}
$$




$$
\begin{aligned}
\frac{d \psi}{d z}= & \frac{k_{m n} e}{r_{c} p_{z} c} \operatorname{Re} \sum_{s=-\infty}^{\infty}\left\{-\frac{\gamma}{\Omega_{e}}\left[\omega f(z)+\frac{i p_{z}}{\gamma m_{e}} f^{\prime}(z)\right]\right. \\
& \cdot \frac{s-m}{k_{m n} r_{c}} J_{s-m}\left(k_{m n} r_{c}\right) J_{s}\left(k_{m n} r_{L}\right) e^{-i A_{s}} \\
& +\frac{k_{m n} r_{L}}{2} f(z)\left[J_{s-1-m}\left(k_{m n} r_{c}\right) J_{s-1}\left(k_{m n} r_{L}\right)\right. \\
& \left.\left.+J_{s+1-m}\left(k_{m n} r_{c}\right) J_{s+1}\left(k_{m n} r_{L}\right)\right] e^{-i A_{s}}\right\} \\
& -\frac{1}{2} \frac{\Omega_{e}^{\prime}}{\Omega_{e}} \frac{r_{L}}{r_{c}} \cos (\varphi-\psi) \\
\frac{d t}{d z}= & \frac{\gamma m_{e}}{p_{z}} \\
\frac{d p_{\perp}}{d z}= & \frac{\gamma m_{e} e}{p_{z} c} k_{m n} \operatorname{Re}\left\{i\left[\omega f(z)+\frac{i p_{z}}{\gamma m_{e}} f^{\prime}(z)\right]\right. \\
& \left.\cdot \sum_{s=-\infty}^{\infty} J_{s-m}\left(k_{m n} r_{c}\right) J_{s}^{\prime}\left(k_{m n} r_{L}\right) e^{-i A_{s}}\right\} \\
& +\frac{1}{2} m_{e} \Omega_{e}^{\prime}\left[r_{L}+r_{c} \sin (\varphi-\psi)\right] \\
\frac{d \varphi}{d z}= & \frac{\gamma m_{e} e}{p_{z} p_{\perp} c} k_{m n} \sum_{s=-\infty}^{\infty} \operatorname{Re}\left\{\left[-\frac{s}{k_{m n} r_{L}}\right.\right. \\
& \left.\cdot\left[\omega f(z)+\frac{i p_{z}}{\gamma m_{e}} f^{\prime}(z)\right]+\frac{k_{m n} p_{\perp}}{\gamma m_{e}} f(z)\right] \\
& \left.\cdot J_{s-m}\left(k_{m n} r_{c}\right) J_{s}\left(k_{m n} r_{L}\right) e^{-i A_{s}}\right\} \\
& +\frac{m_{e} \Omega_{e}}{p_{z}}+\frac{m_{e} \Omega_{e}^{\prime}}{2 p_{\perp}} r_{c} \cos (\varphi-\psi) 1 \\
& {[\mathrm{~A} .11} \\
&
\end{aligned}
$$

and

$$
\begin{aligned}
\frac{d p_{z}}{d z}= & \frac{e}{c} \frac{p_{\perp}}{p_{z}} k_{m n} \operatorname{Re}\left[f^{\prime}(z) \sum_{s=-\infty}^{\infty} J_{s-m}\left(k_{m n} r_{c}\right)\right. \\
& \left.\cdot J_{s}^{\prime}\left(k_{m n} r_{L}\right) e^{-i A_{s}}\right] \\
& -\frac{m_{e} \Omega_{e}^{\prime} p_{\perp}}{2 p_{z}}\left[r_{L}+r_{c} \sin (\varphi-\psi)\right]
\end{aligned}
$$

where $A_{s}$ for the $j$ th electron is defined in (A.5).

Equations (A.4) and (A.11) form a set of coupled equations in slow variables for the fields and the electrons. Conditions of the gyrotron are usually tuned to favor interaction at a particular cyclotron harmonic numbers $s$, such that $A_{s}$ is a slowly varying function of $z$. Then only one term in the summation is significant while the other terms will be averaged out upon integration in $z$.

\section{ACKNOWLEDGMENT}

The authors wish to thank Prof. Neville C. Luhmann, Jr., for many stimulating discussions.

\section{REFERENCES}

[1] J. L. Seftor, V. L. Granatstein, K. R. Chu, P. Sprangle, and M. E. Read, "The electron cyclotron cyclotron maser as a high power traveling-wave amplifier of millimeter waves," IEEE J. Quantum Electron., vol. 15, pp. 848-853, 1979.

[2] L. R. Barnett, K. R. Chu, J. M. Baird, V. L. Granatstein, and A. T. Drobot, "Gain, saturation, and bandwidth measurements of the NRL gyrotron traveling wave amplifier," in Proc. Tech. Dig. Int. Electron Devices Meeting. New York: IEEE, 1979, pp. 164-167.

[3] L. R. Barnett, J. M. Baird, Y. Y. Lau, K. R. Chu, and V. L. Granatstein, "A high gain single stage gyrotron traveling-wave amplifier," in Proc. Tech. Dig. Int. Electron Devices Meeting, New York: IEEE, 1980, pp. 314-317.

[4] R. S. Symons, H. R. Jory, S. J. Hegji, and P. E. Ferguson, "An experimental Gyro-TWT," IEEE Trans. Microwave Theory Tech., vol. 29, pp. 181-184, 1981.

[5] P. E. Ferguson, G. Valier, and R. S. Symons, "Gyrotron-TWT operating characteristics," IEEE Trans. Microwave Theory Tech., vol. 29, pp. 794-799, 1981

[6] L. R. Barnett, L. H. Chang, H. Y. Chen, K. R. Chu, Y. K. Lau, and C. C. Tu, "Absolute instability competition and suppression in a millimeter-wave gyrotron traveling-wave tube," Phys. Rev. Lett., vol. 63, pp. 1062-1065, 1989.

[7] K. R. Chu, L. R. Barnett, W. K. Lau, L. H. Chang, and H. Y. Chen, "A wide-band millimeter-wave gyrotron traveling-wave amplifier experiment," IEEE Trans. Electron Devices, vol. 37, pp. 1557-1560, 1990.

[8] K. R. Chu, L. R. Barnett, W. K. Lau, L. H. Chang, and C. S. Kou, "Recent development in millimeter wave Gyro-TWT research at NTHU," in Proc. Tech. Dig. Int. Electron Devices Meeting. New York: IEEE, 1990, pp. 699-702.

[9] G. S. Park, S. Y. Park, R. H. Kyser, A. K. Ganguly, and C. M. Armstrong, "Gain broadening in an inhomogeneous gyrotron traveling wave amplifier," in Proc. Tech. Dig. Int. Electron Device Meeting. New York: IEEE, 1990, pp. 703-705 and 1991, pp. 779-781.

[10] G. S. Park, J. J. Choi, S. Y. Park, C. M. Armstrong, A. K. Ganguly, and R. H. Kyser, "Broadband operation of a two-stage tapered gyroTWT amplifier," in Proc. Tech. Dig. Int. Electron Device Meeting. New Jersey: IEEE, 1993, pp. 351-353.

[11] Q. S. Wang, K. C. Leou, C. K. Chong, A. J. Balkcum, D. B. McDermott, and N. C. Luhmann, Jr., "Gyro-TWT amplifier development at UCD," in Proc. Dig. 19th Int. Conf. Infrared and Millimeter Waves, K. Sakai and T. Yoneyama, Eds. Sendai, Japan, Oct. 1994, pp. 415-416.

[12] K. C. Leou, D. B. McDermott, A. J. Balkcum, and N. C. Luhmann, Jr., "Stable high power TE 01 gyro-TWT amplifiers," IEEE Trans. Plasma Sci., vol. 22, pp. 585-592, 1994.

[13] K. R. Chu, L. R. Barnett, H. Y. Chen, S. H. Chen, Ch. Wang, Y. S. Yeh, Y. C. Tsai, T. T. Yang, and T. Y. Dawn, "Stabilizing of absolute instabilities in gyrotron traveling-wave amplifier," Phys. Rev., Lett., vol. 74, pp. 1103-1106, 1995.

[14] Q. S. Wang, D. B. McDermott, and N. C. Luhmann, Jr. "Demonstration of marginal stability theory by a $200-\mathrm{kW}$ second-harmonic gyro-TWT amplifier," Phys. Rev. Lett., vol. 75, pp. 4322-4325, 1995.

[15] G. S. Park, J. J. Choi, S. Y. Park, C. M. Armstrong, A. K. Ganguly, R. H. Kyser, and R. K. Parker, "Gain broadening of two-stage tapered gyrotron traveling wave tube amplifier," Phys. Rev. Lett., vol. 74, pp. 2399-2402, 1995.

[16] V. L. Granatstein, B. Levush, B. G. Danly, and R. K. Parker, "A quarter century of gyrotron research and development," IEEE Trans. Plasma Sci., vol. 25, pp. 1322-1335, 1997.

[17] K. R. Chu, H. Y. Chen, C. L. Hung, T. H. Chang, L. R. Barnett, S H. Chen, and T. T. Yang, "Ultra high gain gyrotron traveling wave amplifier," Phys. Rev. Lett., vol. 81, pp. 4760-4763, 1998.

[18] Y. Y. Lau, K. R. Chu, L. R. Barnett, and V. L. Granatstein, "Gyrotron traveling wave amplifier: I. Analysis of oscillations," Int. J. Infrared Millimeter Waves, vol. 2, pp. 373-393, 1981.

[19] A. T. Lin, K. R. Chu, and A. Abromborsky, "Stability and tunability of a CARM amplifier," IEEE Trans. Electron Devices, vol. 34, pp. 2621-2624, 1987.

[20] K. R. Chu and A. T. Lin, "Gain and bandwidth of the Gyro-TWT and CARM amplifier," IEEE Trans. Plasma Sci., vol. 16, pp. 90-104, 1988.

[21] J. A. Davies, "Conditions for absolute instability in the cyclotron resonance maser," Phys. Fluids B, vol. 1, pp. 663-669, 1989.

[22] A. T. Lin, C. C. Lin, and K. R. Chu, "Stability of a high order mode CARM amplifier," IEEE Trans. Electron Devices, vol. 36, pp. 785-788, 1989.

[23] A. T. Lin and C. C. Lin, "Stabilization of the absolute instability in cyclotron autoresonance maser amplifiers by a drive wave," Phys. Fluids $B$, vol. 1, pp. 2286-2288, 1989.

[24] K. R. Chu, L. R. Barnett, W. K. Lau, L. H. Chang, A. T. Lin, and C. C. Lin, "Nonlinear dynamics of the gyrotron traveling-wave amplifier," Phys. Fluids, vol. B3, pp. 2403-2408, 1991.

[25] A. T. Lin, K. R. Chu, C. C. Lin, C. S. Kou, D. B. McDermott, and N. C. Luhmann. Jr. "Marginal stability design criterion for Gyro-TWT's and comparison of fundamental with second harmonic operation," Int. 
J. Electron. vol. 72, pp. 873-885, 1992.

[26] P. Sprangle and W. M. Manheimer, "Coherent nonlinear theory of a cyclotron instability," Phys. Fluids, vol. 18, pp. 224-230, 1975.

[27] P. Sprangle and A. T. Drobot, "The linear and self-consistent nonlinear theory of the electron cyclotron maser instability," IEEE Trans. Microwave Theory Tech., vol. 25, pp. 528-544, 1977.

[28] K. R. Chu, A. T. Drobot, H. H. Szu, and P. Sprangle, "Theory and simulation of the gyrotron traveling wave amplifier operating at cyclotron harmonics," IEEE Trans. Microwave Theory Tech., vol. 28, pp. 313-317, 1980 .

[29] A. K. Ganguly and S. Ahn, "Self-consistent large theory of the gyrotron traveling wave amplifier," Int. J. Electron., vol. 53, pp. 641-658, 1982.

[30] _ "Large-signal theory of a two-stage wide band Gyro-TWT," IEEE Trans. Electron Devices, vol. 31, pp. 474-480, 1984.

[31] A. W. Fliflet, "Linear and nonlinear theory of the doppler-shifted cyclotron resonance maser based on TE and TM waveguide modes," Int. J. Electron., vol. 61, pp. 1049-1080, 1986

[32] C. S. Kou, Q. S. Wang, D. B. McDermott, A. T. Lin, K. R. Chu, and N. C. Luhmann, Jr., "High-power harmonics Gyro-TWT Part I: Linear theory and oscillation study," IEEE Trans. Plasma Sci., vol. 20, pp. 155-162, 1992.

[33] Y. Y. Lau, K. R. Chu, L. R. Barnett, and V. L. Granatstein, "Effects of velocity spread and wall resistivity on the gain and bandwidth of the gyrotron traveling-wave amplifier," Int. J. Infrared Millimeter Waves, vol. 2, pp. 395-413, 1981

[34] Ch. Wang, Y. S. Yeh, T. T. Yang, H. Y. Chen, S. H. Chen, Y. C. Tsai, L. R. Barnett, and K. R. Chu, "A mechanically tunable magnetron injection gun,” Rev. Sci. Instrum., vol. 68, pp. 3031-3035, 1997.

[35] T. H. Chang, L. R. Barnett, K. R. Chu, F. Tai, and C. L. Hsu, "A dual-function circular polarization converter for microwave/plasma processing systems," Rev. Sci. Instrum., vol. 70, pp. 1530-1534, 1999.

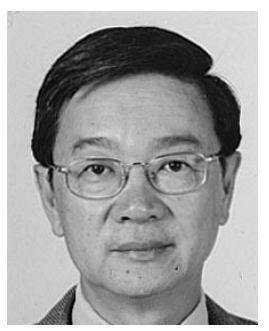

Kwo Ray Chu (SM'82-F'97) received the B.S. degree in physics from National Taiwan University, R.O.C., in 1965, the M.S. degree in physics from the University of Massachusetts, Amherst, in 1968 , and the Ph.D. degree in applied physics from Cornell University, Ithaca, NY, in 1972. His fields of expertise include plasma physics, electromagnetics, and relativistic electronics.

From 1973 to 1983 , he served in the High Power Electromagnetic Radiation Branch of the U.S. Naval Research Laboratory, Washington, DC, where he headed the Advanced Concepts Section from 1980 to 1983 conducting research on relativistic electronics with emphasis on coherent electromagnetic radiation generation, while concurrently serving as Adjunct Associate Professor in the Department of Applied Sciences at Yale University, New Haven, CT. Since September 1983, he has been Professor of Physics in National Tsing Hua University, Taiwan, R.O.C. In 1997, he was awarded the title of National Chair by the Ministry of Education, R.O.C.

Dr. Chu was elected Fellow of the American Physical Society in 1983.

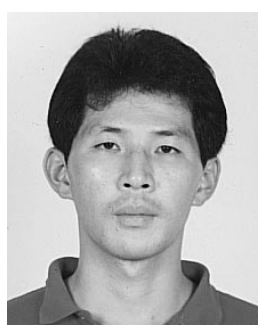

Han-Ying Chen was born in Hualien, Taiwan, R.O.C., on May 31, 1964. He received the B.S degree in electrophysics from National Chiao Tung University, R.O.C., in 1986 and the M.S. and Ph.D. degrees in physics from National Tsing Hua University, R.O.C., in 1989 and 1996, respectively.

Upon graduation, he became a postdoctoral fellow working on novel high-power RF sources in the Physics Department of National Tsing Hua University, R.O.C., for two years. He currently serves as Design Engineer in the Microwave Technology Department Division of Electronics Research and Service Organization, Industrial Technology Research Institute, Hsinchu, Taiwan. His principal responsibility is concerned with the research and development of microwave tubes.

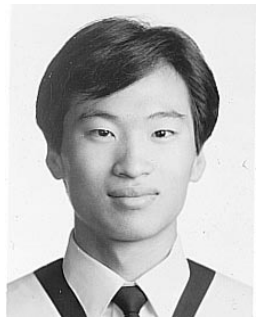

Chien-Lung Hung was born in Peng-hu, Taiwan, R.O.C., 1970. He received the B.S. degree in physics from National Changhua University of Education in 1992, and the Ph.D. degree in physics from National Tsing Hua University, R.O.C., in 1998. His Ph.D. thesis research was on high gain gyro-TWT and complex RF structures. He currently is in the military service.

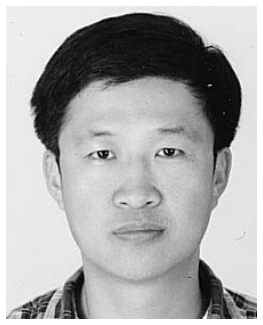

Tsun-Hsu Chang received the B.S. degree in physics from National Central University in 1991. $\mathrm{He}$ is currently pursuing the Ph.D. degree in the Physics Department of National Tsing Hua University, R.O.C.

After two years of military service, he served for one year as Research Assistant in the accelerator facility of the Institute of Physics, Academia Sinica. His research interests include high-power microwave/millimeter wave generation, large-area uniform density plasma generation and diagnostics. His current research involves theoretical and experimental studies of spurious oscillations in gyro-TWT and fundamental physics issues of gyro-BWO. $\mathrm{He}$ also designs couplers/mode converters for high-order mode gyrotron experiments.

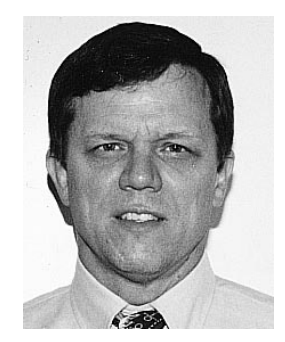

Larry R. Barnett (M'78) received the B.S. degree in electrical engineering from Tennessee Technological University, Cookeville, in 1972, the M.S.E.E degree from the University of Tennessee Space Institute in 1975, and the Ph.D. degree in electrical engineering from the University of Tennessee Nashville, in 1978.

From 1978 to 1983 , he was at the U.S. Naval Research Laboratory, Washington, DC, in the High Power Electromagnetic Radiation Branch, where he worked on gyratron devices. From 1983 to 1989 , he was an Associate Research Professor at the University of Utah, Salt Lake City, working on submillimeter-wave BWO's and gyrotron devices. Since 1989, he has been a consultant for microwave tubes, microwave devices, high-voltage power supplies, and transmitters. He serves as a visiting expert to National Tsing Hua University, Taiwan, on millimeter-wave gyrotron traveling wave amplifiers. His founder of Mountain Technology, a small company that does prototype development of specialized power supplies, transmitters, and microwave devices.

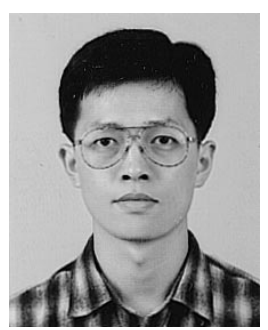

Shih-Huang Chen was born in Taiwan, R.O.C., in 1967. He received the B.S. and Ph.D. degrees from the Department of Nuclear Engineering, National Tsing Hua University, R.O.C., in 1989 and 1995, respectively. From 1990 to 1995 , he conducted Ph.D. research on gyrotron devices in the High Frequency Electromagnetic Laboratory of the Department of Physics, National Tsing Hua University. He joined the National Center for High-performance Computing as Associate Research Scientist in October 1995. His current research interests include computational plasma physics, high power microwave sources, and electron optics. 


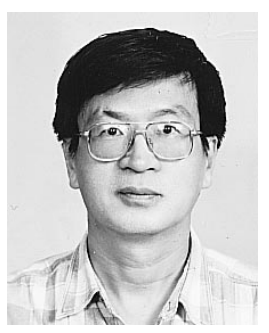

Tz-Te Yang received from the degree in mechanical engineering from $\mathrm{Da}$ Hua Technical College, R.O.C., in 1973.

He currently serves as an Assistant Engineer in the RF Group of the Synchrotron Radiation Research Center, Hsinchu, Taiwan, R.O.C. His principle responsibility includes engineering design and fabrication of RF cavities, tuners and vacuum windows for the storage ring, RF gun, and microwave/millimeter wave tubes.
Demosthenes J. Dialetis received the B.S. degree from University of Athens, Greece, in 1960, the Ph.D. degree from University of Rochester, NY, in 1967, and the M.S. degree from Duke University, Durham, NC, in 1976.

$\mathrm{He}$ has more than 25 years of experience in theoretical and engineering physics. His specific research interests have included high-power microwave devices and high-power compact cyclic accelerators, coherent phenomena in the interaction of radiation with matter, photon counting statistics, and the theory of communication systems. Since 1979, he has pursued these research interests at Science Applications International Corporation (SAIC). Before that, he was employed as a Numerical Analyst at Computer Sciences Corporation, and as a Research Physicist at the U.S. Army Research Office. As a Research Physicist at SAIC, has conducted research related to the development of cross-field amplifiers (CFA's), high-current cyclic induction accelerators, high-power gyrotron oscillators and gyrotron traveling wave amplifiers. Specifically, he has studies the multimode operation of the gyrotron device, design high-power gyrotron oscillators with complex cavity structure, and formulated the linear and nonlinear theory of the gyrotron traveling wave amplifier, including a scheme for side-band amplification. He has developed a refined model of anode circuit in CFA that takes into account the nonlinear behavior of the potential at the boundary between the vanes. By appropriate modification of the basic network in the anode circuit of the CFA, he showed that it is possible to match its specific characteristics much better than before. $\mathrm{He}$ has also provided analytical and numerical theory support for projects on antenna design and development of a new class of electromagnetic sensors. 